MATHEMATICS OF COMPUTATION

S 0025-5718(99)01146-1

Article electronically published on August 17, 1999

\title{
FINITE ELEMENT APPROXIMATION FOR EQUATIONS OF MAGNETOHYDRODYNAMICS
}

\author{
MATTHIAS WIEDMER
}

\begin{abstract}
We consider the equations of stationary incompressible magnetohydrodynamics posed in three dimensions, and treat the full coupled system of equations with inhomogeneous boundary conditions. We prove the existence of solutions without any conditions on the data. Also we discuss a finite element discretization and prove the existence of a discrete solution, again without any conditions on the data. Finally, we derive error estimates for the nonlinear case.
\end{abstract}

\section{INTRODUCTION}

In this work we study the equations of stationary incompressible magnetohydrodynamics which describe the flow of an electric conducting material under the influence of a magnetic field. We treat the equations in a three-dimensional domain and with the same inhomogeneous boundary conditions as in [9]. In contrast with the results in [9], we derive the existence of solutions of both continuous and discrete problems without any conditions on the boundary data of the velocity. We also obtain an error estimate for the general nonlinear case, and not only for the case where the solutions of the continuous and discrete problem are unique.

The main problem for this type of nonlinear equation with inhomogeneous boundary conditions is the fact that the corresponding homogeneous problem differs from the original problem not only in the right-hand side, but also in the nonlinear form on the left-hand side. This form contains additional terms with the continuation of the boundary conditions, which cause problems in the proof of the coercivity. We overcome this problem by the construction of suitable continuations of the boundary data. To obtain an error estimate in the general nonlinear case we reformulate the given equations as an operator problem which fits into the abstract framework of [2], and derive the error estimate by methods similar to those in [5] or [11].

The paper is organized as follows. In Section 2 we introduce some function spaces that are needed throughout. Section 3 is concerned with the presentation of the equations and the derivation of the weak formulation and the homogeneous problem. In Section 4 we construct a suitable continuation of the boundary data for the velocity, and derive an existence result without any conditions on the data. Section 5 deals with the finite element approximation of the equations, and we prove existence of solutions, again without conditions on the data. In Section 6 we

Received by the editor January 19, 1998.

1991 Mathematics Subject Classification. Primary 65N30, 65N15, 76W05, 35Q20.

Key words and phrases. Magnetohydrodynamics, nonlinear problems, inhomogeneous boundary conditions. 
reformulate the equations of magnetohydrodynamics as an operator problem and derive an error estimate for the general nonlinear case.

\section{Notation}

Let $\Omega$ be a bounded domain with boundary $\Gamma:=\partial \Omega$ of class $C^{1,1}$. We denote by $W^{k, p}(\Omega)$ and $H^{k}(\Omega)$ the standard Lebesgue and Sobolev spaces equipped with norms $\|v\|_{W^{k, p}(\Omega)},\|v\|_{k, \Omega}$ and seminorms $|v|_{W^{k, p}(\Omega)},|v|_{k, \Omega}$ (see [1]). We use the notation $\mathbf{H}^{k}(\Omega)$ for vector-valued functions $\mathbf{v}: \Omega \rightarrow \mathbb{R}^{3}$ with components in $H^{k}(\Omega)$; the norm on $\mathbf{H}^{k}(\Omega)$ is given by the canonical Euclidean norm. For our purposes we need two subspaces of $\mathbf{H}^{1}(\Omega)$ that satisfy specific boundary conditions:

$$
\begin{aligned}
\mathbf{H}_{0}^{1}(\Omega) & :=\left\{\mathbf{v} \in \mathbf{H}^{1}(\Omega):\left.\mathbf{v}\right|_{\Gamma}=0\right\}, \\
\mathbf{H}_{n}^{1}(\Omega) & :=\left\{\boldsymbol{\Psi} \in \mathbf{H}^{1}(\Omega):\left.(\boldsymbol{\Psi} \cdot \mathbf{n})\right|_{\Gamma}=0\right\} .
\end{aligned}
$$

We also make use of the product spaces

$$
\begin{aligned}
\mathcal{W}(\Omega) & :=\mathbf{H}^{1}(\Omega) \times \mathbf{H}^{1}(\Omega), \\
\mathcal{W}_{0 n}(\Omega) & :=\mathbf{H}_{0}^{1}(\Omega) \times \mathbf{H}_{n}^{1}(\Omega), \\
\mathcal{W}_{\mathbf{g} q}(\Omega) & :=\left\{\mathbf{v} \in \mathbf{H}^{1}(\Omega): \mathbf{v}=\mathbf{g} \text { on } \Gamma\right\} \times\left\{\boldsymbol{\Psi} \in \mathbf{H}^{1}(\Omega): \mathbf{\Psi} \cdot \mathbf{n}=q \text { on } \Gamma\right\}
\end{aligned}
$$

with the usual norm

$$
\|(\mathbf{v}, \mathbf{\Psi})\|_{\mathcal{W}}:=\left(\|\mathbf{v}\|_{1, \Omega}^{2}+\|\boldsymbol{\Psi}\|_{1, \Omega}^{2}\right)^{1 / 2} .
$$

Next we define

$$
\mathbf{Z}(\Omega):=\left\{\mathbf{v} \in \mathbf{H}_{0}^{1}(\Omega): \nabla \cdot \mathbf{v}=0\right\}
$$

which is a subspace of the solenoidal functions, and a subspace of $L^{2}(\Omega)$

$$
L_{0}^{2}(\Omega):=\left\{q \in L^{2}(\Omega): \int_{\Omega} q=0\right\}
$$

the space of $L^{2}$-functions with zero mean value on $\Omega$.

We also need the notation $\mathbf{H}^{-1}(\Omega)$ for the dual space of $\mathbf{H}_{0}^{1}(\Omega)$, and by $\langle\cdot, \cdot\rangle_{\Omega}$ we denote the duality pairing between these spaces. The norm of $\mathbf{H}^{-1}(\Omega)$ is defined as usual:

$$
\|\mathbf{f}\|_{-1, \Omega}:=\sup _{\substack{\mathbf{v} \in \mathbf{H}_{0}^{1}(\Omega) \\ \mathbf{v} \neq 0}} \frac{\langle\mathbf{f}, \mathbf{v}\rangle_{\Omega}}{|\mathbf{v}|_{1, \Omega}} .
$$

For the boundary conditions we make use of the trace space

$$
H^{1 / 2}(\Gamma):=\left\{\left.v\right|_{\Gamma}: v \in H^{1}(\Omega)\right\}
$$

and analogously for vector-valued functions $\mathbf{H}^{1 / 2}(\Gamma)$. We denote their duals by $H^{-1 / 2}(\Gamma)$ and $\mathbf{H}^{-1 / 2}(\Gamma)$, and the duality pairing by $\langle\cdot, \cdot\rangle_{\Gamma}$. The norms in these spaces are defined in a canonical manner (see [9]). 


\section{EQUATIONS AND THE WEAK FORMULATION}

We consider the equations of stationary, incompressible magnetohydrodynamics in a domain $\Omega \in \mathbb{R}^{3}$ with the described properties. The equations are given in reduced form, i.e., the electric field $\mathbf{E}$ and the current density $\mathbf{j}$ are already eliminated from the original set of equations (for a detailed description of the origin of these equations see [12] and the references therein). The equations with the associated boundary conditions are the following:

$$
\left\{\begin{array}{r}
-\frac{1}{M^{2}} \Delta \mathbf{u}+\frac{1}{N}(\mathbf{u} \cdot \nabla) \mathbf{u}+\nabla p-\frac{1}{R_{m}}(\nabla \times \mathbf{B}) \times \mathbf{B}=\mathbf{f} \\
\nabla \cdot \mathbf{u}=0 \\
\frac{1}{R_{m}} \nabla \times(\nabla \times \mathbf{B})-\nabla \times(\mathbf{u} \times \mathbf{B})=0 \\
\nabla \cdot \mathbf{B}=0
\end{array}\right\} \text { in } \Omega
$$

Here $\mathbf{u}$ denotes the velocity, $\mathbf{B}$ the magnetic field, and $p$ the pressure. All variables have been nondimensionalized (see [9]). For the definition of the parameters $M, N$, and $R_{m}$, which mean the Hartmann number, the interaction parameter, and the magnetic Reynolds number, respectively, we refer again to [12] and the references therein. The function $\mathbf{f}$ belongs to $\mathbf{H}^{-1}(\Omega)$. In order to allow a solution to system (3.1) the following regularity and compability conditions for the boundary data are needed:

$$
\begin{gathered}
\mathbf{g} \in \mathbf{H}^{1 / 2}(\Gamma) \quad \text { with } \int_{\Gamma} \mathbf{g} \cdot \mathbf{n}=0 \\
q \in H^{1 / 2}(\Gamma) \quad \text { with } \int_{\Gamma} q=0 \\
\mathbf{k} \in \mathbf{H}^{-1 / 2}(\Gamma) \quad \text { with } \mathbf{k} \cdot \mathbf{n}=0,\langle\mathbf{k}, 1\rangle_{\Gamma}=0,\langle\mathbf{k}, \nabla \Phi\rangle_{\Gamma}=0 \forall \Phi \in H^{2}(\Omega)
\end{gathered}
$$

Remark 3.1. In problem (3.1) we have no Lagrange multiplier for the condition $\nabla \cdot \mathbf{B}=0$ from the physics. We will see that the system is nevertheless solvable because of the Helmholtz splitting of the space $\mathbf{L}^{2}(\Omega)$ (see Remark 3.4).

Next we present the weak formulation of problem (3.1). For this reason we test the first equation of $(3.1)$ with $\mathbf{v} \in \mathbf{H}_{0}^{1}(\Omega)$, the second with $\chi \in L_{0}^{2}(\Omega)$, and the remaining two equations with $\boldsymbol{\Psi} \in \mathbf{H}_{n}^{1}(\Omega)$. For use in Section 6 we also scale the equations and then obtain the following weak formulation (for details see [12]):

$$
\left\{\begin{array}{cl}
\text { Find }(\mathbf{u}, \mathbf{B}) \in \mathcal{W}_{\mathbf{g} q}(\Omega), p \in L_{0}^{2}(\Omega) \text { such that } \\
a((\mathbf{u}, \mathbf{B}),(\mathbf{u}, \mathbf{B}),(\mathbf{v}, \mathbf{\Psi}))+b((\mathbf{v}, \boldsymbol{\Psi}), p)=F((\mathbf{v}, \mathbf{\Psi})) & \forall(\mathbf{v}, \mathbf{\Psi}) \in \mathcal{W}_{0 n}(\Omega) \\
b((\mathbf{u}, \mathbf{B}), \chi)=0 & \forall \chi \in L_{0}^{2}(\Omega) .
\end{array}\right.
$$


Here the various forms are defined as follows:

$$
\begin{aligned}
& a_{0}((\mathbf{v}, \boldsymbol{\Psi}),(\mathbf{w}, \boldsymbol{\Phi})) \\
& :=\int_{\Omega} \nabla \mathbf{v} \cdot \nabla \mathbf{w}+\int_{\Omega}\{(\nabla \times \mathbf{\Psi}) \cdot(\nabla \times \mathbf{\Phi})+(\nabla \cdot \mathbf{\Psi})(\nabla \cdot \mathbf{\Phi})\}, \\
& a_{1}((\mathbf{u}, \mathbf{B}),(\mathbf{v}, \mathbf{\Psi}),(\mathbf{w}, \mathbf{\Phi})) \\
& :=\frac{M^{2}}{N} \int_{\Omega}[(\mathbf{u} \cdot \nabla) \mathbf{v}] \cdot \mathbf{w}-R_{m} \int_{\Omega}\{[(\nabla \times \mathbf{\Psi}) \times \mathbf{B}] \cdot \mathbf{w}-[(\nabla \times \mathbf{\Phi}) \times \mathbf{B}] \cdot \mathbf{v}\}, \\
& a((\mathbf{u}, \mathbf{B}),(\mathbf{v}, \boldsymbol{\Psi}),(\mathbf{w}, \boldsymbol{\Phi})):=a_{0}((\mathbf{v}, \boldsymbol{\Psi}),(\mathbf{w}, \boldsymbol{\Phi}))+a_{1}((\mathbf{u}, \mathbf{B}),(\mathbf{v}, \boldsymbol{\Psi}),(\mathbf{w}, \boldsymbol{\Phi})) \\
& b((\mathbf{v}, \Psi), \chi):=-\int_{\Omega}(\nabla \cdot \mathbf{v}) \chi, \\
& F((\mathbf{v}, \Psi)):=M^{2} \int_{\Omega} \mathbf{f} \cdot \mathbf{v}+M\left\langle\mathbf{k},\left.\Psi\right|_{\Gamma}\right\rangle_{\Gamma}
\end{aligned}
$$

Remark 3.2. A useful property of the nonlinear form $a_{1}$ is the so-called antisymmetry condition, i.e., for $(\mathbf{u}, \mathbf{B}),(\mathbf{v}, \boldsymbol{\Psi}),(\mathbf{w}, \boldsymbol{\Phi}) \in \mathcal{W}(\Omega)$ with $\nabla \cdot \mathbf{u}=0$, and at least one element of $\{\mathbf{u}, \mathbf{v}, \mathbf{w}\}$ from $\mathbf{H}_{0}^{1}(\Omega)$, the form $a_{1}$ is antisymmetric with respect to its last two arguments:

$$
a_{1}((\mathbf{u}, \mathbf{B}),(\mathbf{v}, \boldsymbol{\Psi}),(\mathbf{w}, \boldsymbol{\Phi}))=-a_{1}((\mathbf{u}, \mathbf{B}),(\mathbf{w}, \boldsymbol{\Phi}),(\mathbf{v}, \boldsymbol{\Psi})) .
$$

Remark 3.3. The equivalence of the weak formulation (3.5) and the system (3.1) was shown in [9]. The main tool there is that for every function $\mathbf{B} \in \mathbf{H}^{1}(\Omega)$ there exists a scalar function $b \in H^{2}(\Omega)$ with $\nabla \cdot \nabla b=\nabla \cdot \mathbf{B}$ and $\left.(\nabla b \cdot \mathbf{n})\right|_{\Gamma}=0$.

Remark 3.4. Note that in (3.5) the condition $\nabla \cdot \mathbf{B}=0$ is not formulated with the introduction of a Lagrange multiplier but with the term $\int_{\Omega}(\nabla \times \mathbf{B}) \cdot(\nabla \times \mathbf{\Psi})$. It is then guaranteed with the compability condition (3.4) on $\mathbf{k}$ and the identity $\nabla \times(\nabla \times b)=0$ for all $b \in H^{2}(\Omega)$.

To conclude this section, we reduce problem (3.5) to a problem with homogeneous boundary conditions. We again use a result from [9] that allows us to split the velocity into a sum of a function that satisfies the given inhomogeneous boundary condition and a function that satisfies homogeneous boundary conditions. The same can be done for the magnetic field. We set

$$
\begin{array}{r}
\mathbf{u}:=\mathbf{u}_{0}+\hat{\mathbf{u}}, \quad \mathbf{u}_{0} \in \mathbf{H}^{1}(\Omega) \text { with } \mathbf{u}_{0}=\mathbf{g} \text { on } \Gamma \text { and } \nabla \cdot \mathbf{u}_{0}=0 \text { in } \Omega, \hat{\mathbf{u}} \in \mathbf{H}_{0}^{1}(\Omega), \\
\mathbf{B}:=\mathbf{B}_{0}+\widehat{\mathbf{B}}, \quad \mathbf{B}_{0} \in \mathbf{H}^{1}(\Omega) \text { with } \mathbf{B}_{0} \cdot \mathbf{n}=q \text { on } \Gamma \\
\quad \text { and } \nabla \cdot \mathbf{B}_{0}=\nabla \times \mathbf{B}_{0}=0, \widehat{\mathbf{B}} \in \mathbf{H}_{n}^{1}(\Omega),
\end{array}
$$

and so obtain the following problem:

$$
\left\{\begin{aligned}
\text { Find }(\hat{\mathbf{u}}, \widehat{\mathbf{B}}) \in \mathcal{W}_{0 n}(\Omega), p \in L_{0}^{2}(\Omega) \text { such that } & \\
\hat{a}((\hat{\mathbf{u}}, \widehat{\mathbf{B}}),(\hat{\mathbf{u}}, \widehat{\mathbf{B}}),(\mathbf{v}, \boldsymbol{\Psi}))+b((\mathbf{v}, \mathbf{\Psi}), p)=\widehat{F}((\mathbf{v}, \boldsymbol{\Psi})) & \forall(\mathbf{v}, \boldsymbol{\Psi}) \in \mathcal{W}_{0 n}(\Omega), \\
b((\hat{\mathbf{u}}, \widehat{\mathbf{B}}), \chi)=0 & \forall \chi \in L_{0}^{2}(\Omega) .
\end{aligned}\right.
$$


Here, $\hat{a}$ and $\widehat{F}$ denote the following forms:

$$
\begin{aligned}
\hat{a}((\mathbf{u}, \mathbf{B}),(\mathbf{v}, \boldsymbol{\Psi}),(\mathbf{w}, \boldsymbol{\Phi})):= & a((\mathbf{u}, \mathbf{B}),(\mathbf{v}, \boldsymbol{\Psi}),(\mathbf{w}, \boldsymbol{\Phi}))+a_{1}\left((\mathbf{v}, \mathbf{\Psi}),\left(\mathbf{u}_{0}, \mathbf{B}_{0}\right),(\mathbf{w}, \boldsymbol{\Phi})\right) \\
& +a_{1}\left(\left(\mathbf{u}_{0}, \mathbf{B}_{0}\right),(\mathbf{v}, \boldsymbol{\Psi}),(\mathbf{w}, \boldsymbol{\Phi})\right), \\
\widehat{F}((\mathbf{v}, \Psi)):= & F((\mathbf{v}, \Psi))-a\left(\left(\mathbf{u}_{0}, \mathbf{B}_{0}\right),\left(\mathbf{u}_{0}, \mathbf{B}_{0}\right),(\mathbf{v}, \mathbf{\Psi})\right) .
\end{aligned}
$$

The key point of Section 4 will be a judicious choice of $\hat{\mathbf{u}}$ and $\widehat{\mathbf{B}}$.

\section{Existence of solutions Without conditions on the DATA}

Problem (3.7) fits into the abstract framework for nonlinear problems described in [5, Chapter IV.1], so we can apply those results. In order to derive an existence result we mainly have to establish - apart from easy properties like the continuity of $\hat{a}$, etc.- - the following properties (see [5]).

1. The trilinear form $\hat{a}$ in problem (3.7) must be coercive, i.e., there exists a constant $\alpha>0$ with

$$
\hat{a}((\mathbf{v}, \Psi),(\mathbf{v}, \Psi),(\mathbf{v}, \Psi)) \geq \alpha \|(\mathbf{v}, \Psi)_{\mathcal{W}}^{2} \quad \forall(\mathbf{v}, \Psi) \in \mathbf{Z}(\Omega) \times \mathbf{H}_{n}^{1}(\Omega) .
$$

2. The bilinear form $b$ has to satisfy the inf-sup condition, i.e., there exists a constant $\beta>0$ with

$$
\inf _{\substack{\chi \in L_{0}^{2}(\Omega) \\ \chi \neq 0}} \sup _{\substack{(\mathbf{v}, \mathbf{\Psi}) \in \mathcal{W}_{0 n}(\Omega) \\(\mathbf{v}, \boldsymbol{\Psi}) \neq 0}} \frac{b((\mathbf{v}, \mathbf{\Psi}), \chi)}{\|(\mathbf{v}, \mathbf{\Psi})\|_{\mathcal{W}}\|\chi\|_{0, \Omega}} \geq \beta .
$$

3. The space $\mathbf{Z}(\Omega) \times \mathbf{H}_{n}^{1}(\Omega)$ must be separable, and the map

$$
(\mathbf{v}, \mathbf{\Psi}) \in \mathbf{Z}(\Omega) \times \mathbf{H}_{n}^{1}(\Omega) \mapsto \hat{a}((\mathbf{v}, \boldsymbol{\Psi}),(\mathbf{v}, \mathbf{\Psi}),(\mathbf{w}, \mathbf{\Phi}))
$$

has to be weakly sequentially continuous on $\mathbf{Z}(\Omega) \times \mathbf{H}_{n}^{1}(\Omega)$, i.e., the following conclusion is valid for all $(\mathbf{w}, \mathbf{\Phi}) \in \mathbf{Z}(\Omega) \times \mathbf{H}_{n}^{1}(\Omega)$ :

$$
\begin{aligned}
& (\mathbf{v}, \Psi)_{m} \underset{m \rightarrow \infty}{\rightarrow}(\mathbf{v}, \Psi) \text { in } \mathbf{Z}(\Omega) \times \mathbf{H}_{n}^{1}(\Omega) \\
& \quad \Rightarrow \hat{a}\left((\mathbf{v}, \Psi)_{m},(\mathbf{v}, \Psi)_{m},(\mathbf{w}, \boldsymbol{\Phi})\right) \underset{m \rightarrow \infty}{\rightarrow} \hat{a}((\mathbf{v}, \Psi),(\mathbf{v}, \Psi),(\mathbf{w}, \boldsymbol{\Phi})) .
\end{aligned}
$$

Condition 2 is the well-known inf-sup condition of the Stokes problem, and property 3 is already established in [9]. Thus it remains to prove the coercivity of $\hat{a}$. In [9], this condition has already been proven, but only with a smallness condition on the boundary data $\mathbf{g}$ for the velocity. This condition can be eliminated if we choose the continuation of the boundary data $\mathbf{g}$ in a suitable manner, as in Lemma 4.3. Before we do this, we need a technical lemma.

Lemma 4.1. Suppose that the domain $\Omega$ has a $C^{1,1}$-boundary $\Gamma$. Then there exists a constant $c:=c(p)>0$ with

$$
\|\phi / d(\cdot, \Gamma)\|_{L^{p}(\Omega)} \leq c|\phi|_{W^{1, p}(\Omega)} \quad \forall \phi \in W_{0}^{1, p}(\Omega), 1<p<\infty .
$$

Here, $d(\cdot, \Gamma)$ denotes the distance from a point $x$ to the boundary $\Gamma$.

Proof. Using the smoothness of $\Gamma$ and introducing a partition of unity, we need only to investigate the case where $\Omega$ is the half-space $\mathbb{R}_{+}^{3}:=\left\{x=\left(x^{\prime}, x_{3}\right) \in \mathbb{R}^{3}: x_{3}>0\right\}$. In this case the function $d$ becomes $d(x, \Gamma)=x_{3}$. Because $C_{0}^{\infty}(\Omega)$ is dense in $L^{p}(\Omega)$ $(1 \leq p<\infty)$, it is sufficient to check that

$$
\int_{\mathbb{R}_{+}^{3}}\left|\phi / x_{3}\right|^{p} d x \leq \tilde{c} \int_{\mathbb{R}_{+}^{3}}\left|\partial \phi / \partial x_{3}\right|^{p} d x \quad \forall \phi \in C_{0}^{\infty}\left(\mathbb{R}_{+}^{3}\right) .
$$


This is an immediate consequence of the Hardy inequality (see [10])

$$
\int_{0}^{\infty}|\phi(t) / t|^{p} d t \leq\left(\frac{p}{p-1}\right)^{p} \int_{0}^{\infty}\left|\phi^{\prime}(t)\right|^{p} d t \quad \forall \phi \in C_{0}^{\infty}((0, \infty)) .
$$

Remark 4.2. We have proven Lemma 4.1 only in the case where $\Omega$ has a $C^{1,1}$ boundary $\Gamma$. Indeed, this is not an essential restriction and we can prove - with more technical effort-Lemma 4.1 (and also Lemma 4.3) for domains which have only a Lipschitz boundary $\Gamma$ (see, e.g. [7]).

We now come to the announced result for the continuation of the boundary data g.

Lemma 4.3. For given $\mathbf{g} \in \mathbf{H}^{1 / 2}(\Gamma)$ with the compability condition (3.2) there exists for any $\varepsilon>0$ a function $\mathbf{u}_{\varepsilon} \in \mathbf{H}^{1}(\Omega)$ with

$$
\begin{gathered}
\mathbf{u}_{\varepsilon}=\mathbf{g} \quad \text { on } \Gamma, \quad \nabla \cdot \mathbf{u}_{\varepsilon}=0 \quad \text { in } \Omega, \\
\left\|\mathbf{u}_{\varepsilon}\right\|_{\mathbf{L}^{3}(\Omega)} \leq \varepsilon\|\mathbf{g}\|_{1 / 2, \Gamma} .
\end{gathered}
$$

Proof. In [5] it is proven that there exists a function $\mathbf{u}_{0} \in \mathbf{H}^{1}(\Omega)$ with

$$
\mathbf{u}_{0}=\mathbf{g} \quad \text { on } \Gamma, \quad \nabla \cdot \mathbf{u}_{0}=0 \quad \text { in } \Omega, \quad\left\|\mathbf{u}_{0}\right\|_{1, \Omega} \leq \gamma_{1}\|\mathbf{g}\|_{1 / 2, \Gamma} .
$$

For this $\mathbf{u}_{0}$ there exists (see [5]) a vector potential $\mathbf{\Psi}_{0} \in \mathbf{H}^{2}(\Omega)$ with the following properties:

$$
\mathbf{u}_{0}=\nabla \times \mathbf{\Psi}_{0}, \quad \boldsymbol{\Psi}_{0} \times \mathbf{n}=0 \quad \text { on } \Gamma, \quad\left\|\boldsymbol{\Psi}_{0}\right\|_{2, \Omega} \leq c\left\|\mathbf{u}_{0}\right\|_{1, \Omega} .
$$

For any $\eta>0$ it is again proven in [5, Lemma IV.2.4] that there exists a function $\theta_{\eta} \in C^{2}(\bar{\Omega})$ with

$$
\begin{aligned}
\theta_{\eta} & =1 & & \text { in a neighborhood of } \Gamma, \\
\theta_{\eta}(x) & =0 & & \text { if } d(x, \Gamma) \geq \delta(\eta):=e^{-1 / \eta}, \\
\partial \theta_{\eta}(x) / \partial x_{i} & \leq \eta / d(x, \Gamma) & & \text { if } d(x, \Gamma) \leq \delta(\eta), 1 \leq i \leq 3 .
\end{aligned}
$$

We construct the function $\mathbf{u}_{\eta} \in \mathbf{H}^{1}(\Omega)$ as follows:

$$
\mathbf{u}_{\eta}:=\nabla \times\left(\theta_{\eta} \mathbf{\Psi}_{0}\right)=\underbrace{\nabla \theta_{\eta} \times \mathbf{\Psi}_{0}}_{=: \mathbf{u}_{\eta, 1}}+\underbrace{\theta_{\eta}\left(\nabla \times \mathbf{\Psi}_{0}\right)}_{=: \mathbf{u}_{\eta, 2}} .
$$

From the construction we easily obtain

$$
\mathbf{u}_{\eta}=\mathbf{g} \quad \text { on } \Gamma \quad \text { and } \quad \nabla \cdot \mathbf{u}_{\eta}=\nabla \cdot\left(\nabla \times\left(\theta_{\eta} \mathbf{\Psi}_{0}\right)\right)=0 .
$$

It remains to prove (4.3). For this purpose we consider the terms $\mathbf{u}_{\eta, 1}$ and $\mathbf{u}_{\eta, 2}$ separately. For $\mathbf{u}_{\eta, 2}$ the following estimate holds:

$$
\begin{aligned}
\left\|\mathbf{u}_{\eta, 2}\right\|_{\mathbf{L}^{3}(\Omega)} & =\left\{\int_{d(x, \Gamma) \leq \delta(\eta)}\left|\mathbf{u}_{\eta, 2}(x)\right|^{3} d x\right\}^{1 / 3} \\
& =\left\{\int_{d(x, \Gamma) \leq \delta(\eta)}\left|\theta_{\eta}(x)\left(\nabla \times \mathbf{\Psi}_{0}(x)\right)\right|^{3} d x\right\}^{1 / 3} \\
& \leq c_{1}(\operatorname{meas}\{x \in \Omega: d(x, \Gamma) \leq \delta(\eta)\})^{1 / 6}\left\|\nabla \times \mathbf{\Psi}_{0}\right\|_{\mathbf{L}^{6}(\Omega)} \\
& \leq c_{2}(\operatorname{meas}\{x \in \Omega: d(x, \Gamma) \leq \delta(\eta)\})^{1 / 6}\left\|\mathbf{\Psi}_{0}\right\|_{2, \Omega} \\
& \leq c_{3}(\operatorname{meas}\{x \in \Omega: d(x, \Gamma) \leq \delta(\eta)\})^{1 / 6}\|\mathbf{g}\|_{1 / 2, \Gamma},
\end{aligned}
$$


where we have used the Sobolev imbedding theorem $\mathbf{H}^{1}(\Omega) \hookrightarrow \mathbf{L}^{6}(\Omega)$, the Hölder inequality and the estimate (4.4).

In order to treat $\mathbf{u}_{\eta, 1}$ we have, as in Lemma 4.1, only to investigate the case where $\Omega$ is the half-space $\Omega:=\left\{\left(x^{\prime}, x_{3}\right) \in \mathbb{R}^{3}: x^{\prime} \in \mathbb{R}^{2}, x_{3}>0\right\}$. Let us consider the function $\theta_{\eta}(x)$ defined by $(\delta(\eta)$ as before):

$$
\theta_{\eta}(x)=\varphi_{\eta}\left(x_{3}\right)= \begin{cases}1 & 0 \leq x_{3} \leq \delta(\eta)^{2} \\ \eta \ln \left(\delta(\eta) / x_{3}\right) & \delta(\eta)^{2} \leq x_{3} \leq \delta(\eta) \\ 0 & \text { otherwise }\end{cases}
$$

For the gradient of $\varphi_{\eta}$ we have

$$
\nabla \varphi_{\eta}=-\frac{\eta}{x_{3}} \mathbf{e}_{3}, \quad \delta(\eta)^{2} \leq x_{3} \leq \delta(\eta)
$$

and

$$
\nabla \varphi_{\eta} \times \mathbf{\Psi}_{0}=\frac{\eta}{x_{3}}\left(\begin{array}{c}
\Psi_{0,2} \\
-\Psi_{0,1} \\
0
\end{array}\right), \quad \delta(\eta)^{2} \leq x_{3} \leq \delta(\eta) .
$$

This implies

$$
\begin{aligned}
\left\|\mathbf{u}_{\eta, 1}\right\|_{\mathbf{L}^{2}(\Omega)} & =\left\|\nabla \varphi_{\eta} \times \mathbf{\Psi}_{0}\right\|_{\mathbf{L}^{3}(\Omega)} \\
& \leq \eta \sum_{i=1}^{2}\left\|\Psi_{0, i}\left(x^{\prime}, x_{3}\right) / x_{3}\right\|_{L^{3}(\Omega)} .
\end{aligned}
$$

Taking (4.5) into account, we can conclude that $\Psi_{0, i}=0$ on $\Gamma(i=1,2)$ and that $\Psi_{0, i} \in H^{2}(\Omega) \cap H_{0}^{1}(\Omega)$. Therefore, using Sobolev's imbedding theorem $W^{2,2}(\Omega) \hookrightarrow$ $W^{1,3}(\Omega)$ (see [1]), we can apply Lemma 4.1 (with $p=3$ ) for $\Psi_{0, i}(i=1,2)$. For $i=1,2$ this yields the estimate

$$
\left\|\Psi_{0, i} / x_{3}\right\|_{L^{3}(\Omega)} \leq c_{4}\left|\Psi_{0, i}\right|_{W^{1,3}(\Omega)} \leq c_{5}\left\|\Psi_{0, i}\right\|_{2, \Omega} \leq c_{6}\|\mathbf{g}\|_{1 / 2, \Gamma} .
$$

Thus, for $\mathbf{u}_{\eta, 1}$ we obtain

$$
\left\|\mathbf{u}_{\eta, 1}\right\|_{\mathbf{L}^{3}(\Omega)} \leq \eta \sum_{i=1}^{2}\left\|\Psi_{0, i} / x_{3}\right\|_{L^{3}(\Omega)} \leq \eta c_{7}\|\mathbf{g}\|_{1 / 2, \Gamma} .
$$

Finally, we may choose $\eta:=\eta(\varepsilon)$ small enough such that

$$
c_{3}(\operatorname{meas}\{x \in \Omega: d(x, \Gamma) \leq \delta(\eta)\})^{1 / 6}+\eta c_{7} \leq \varepsilon .
$$

Then the desired inequality (4.3) follows immediately from (4.6) and (4.7).

With Lemma 4.3 we are now able to establish the coercivity condition (4.1) without any smallness conditions on the boundary data $\mathbf{g}$ of the velocity.

Lemma 4.4. The trilinear form $\hat{a}$ from (3.7) is coercive, i.e., there exists a constant $\alpha>0$ with

$$
\hat{a}((\mathbf{v}, \Psi),(\mathbf{v}, \Psi),(\mathbf{v}, \Psi)) \geq \alpha\|(\mathbf{v}, \Psi)\|_{\mathcal{W}}^{2} \quad \forall(\mathbf{v}, \Psi) \in \mathbf{Z}(\Omega) \times \mathbf{H}_{n}^{1}(\Omega) .
$$


Proof. Using standard results, we infer that the following inequality holds for all $(\mathbf{v}, \mathbf{\Psi}) \in \mathbf{Z}(\Omega) \times \mathbf{H}_{n}^{1}(\Omega)$ (for details see [12]):

$$
\begin{aligned}
& \hat{a}((\mathbf{v}, \boldsymbol{\Psi}),(\mathbf{v}, \boldsymbol{\Psi}),(\mathbf{v}, \boldsymbol{\Psi})) \geq k_{1}\|\mathbf{v}\|_{1, \Omega}^{2}+k_{2}\|\boldsymbol{\Psi}\|_{1, \Omega}^{2} \\
& +\underbrace{\frac{M^{2}}{N} \int_{\Omega}\left[(\mathbf{v} \cdot \nabla) \mathbf{u}_{0}\right] \cdot \mathbf{v}}_{=(1)}+\underbrace{R_{m} \int_{\Omega}[(\nabla \times \boldsymbol{\Psi}) \times \boldsymbol{\Psi}] \cdot \mathbf{u}_{0}}_{=(2)} .
\end{aligned}
$$

Next we treat the terms (1) and (2) in (4.8) separately. For (1) we have, by a generalized Hölder's inequality and the continuous imbedding of $\mathbf{H}^{1}(\Omega)$ in $\mathbf{L}^{6}(\Omega)$,

$$
\begin{aligned}
\left|\frac{M^{2}}{N} \int_{\Omega}\left[(\mathbf{v} \cdot \nabla) \mathbf{u}_{0}\right] \cdot \mathbf{v}\right| & =\left|-\frac{M^{2}}{N} \int_{\Omega}[(\mathbf{v} \cdot \nabla) \mathbf{v}] \cdot \mathbf{u}_{0}\right| \\
& \leq \frac{M^{2}}{N}\|\mathbf{v}\|_{\mathbf{L}^{6}(\Omega)}\|\nabla \mathbf{v}\|_{0, \Omega}\left\|\mathbf{u}_{0}\right\|_{\mathbf{L}^{3}(\Omega)} \\
& \leq c_{1} \frac{M^{2}}{N}\|\mathbf{v}\|_{1, \Omega}^{2}\left\|\mathbf{u}_{0}\right\|_{\mathbf{L}^{3}(\Omega)}
\end{aligned}
$$

Similarly for (2),

$$
\begin{aligned}
\left|R_{m} \int_{\Omega}[(\nabla \times \boldsymbol{\Psi}) \times \boldsymbol{\Psi}] \cdot \mathbf{u}_{0}\right| & \leq R_{m}\|\nabla \times \boldsymbol{\Psi}\|_{0, \Omega}\|\boldsymbol{\Psi}\|_{\mathbf{L}^{6}(\Omega)}\left\|\mathbf{u}_{0}\right\|_{\mathbf{L}^{3}(\Omega)} \\
& \leq c_{2} R_{m}\|\boldsymbol{\Psi}\|_{1, \Omega}^{2}\left\|\mathbf{u}_{0}\right\|_{\mathbf{L}^{3}(\Omega)}
\end{aligned}
$$

Therefore, using Lemma 4.3, we can choose $\mathbf{u}_{0}$ such that along with (4.9) and (4.10) the following inequalities hold:

$$
\begin{aligned}
& (1) \leq \varepsilon\|\mathbf{v}\|_{1, \Omega}^{2}, \\
& (2) \leq \varepsilon\|\boldsymbol{\Psi}\|_{1, \Omega}^{2},
\end{aligned}
$$

with $\varepsilon<\min \left\{k_{1}, k_{2}\right\}$.

Thus, the desired estimate follows from (4.8), (4.11), and (4.12):

$$
\begin{aligned}
\hat{a}((\mathbf{v}, \Psi),(\mathbf{v}, \Psi),(\mathbf{v}, \Psi)) & \geq\left(k_{1}-\varepsilon\right)\|\mathbf{v}\|_{1, \Omega}^{2}+\left(k_{2}-\varepsilon\right)\|\boldsymbol{\Psi}\|_{1, \Omega}^{2} \\
& \geq \alpha\|(\mathbf{v}, \Psi)\|_{\mathcal{W}}^{2} \quad \forall(\mathbf{v}, \Psi) \in \mathbf{Z}(\Omega) \times \mathbf{H}_{n}^{1}(\Omega),
\end{aligned}
$$

with $\alpha:=\min \left\{k_{1}-\varepsilon, k_{2}-\varepsilon\right\}$.

Lemma 4.4 leads to the following existence theorem.

Theorem 4.5. For all functions $\mathbf{f} \in \mathbf{H}^{-1}(\Omega)$ and for all boundary data $\mathbf{g}, q, \mathbf{k}$ which satisfy the regularity and compability assumptions (3.2)-(3.4), problem (3.5) has at least one solution $((\mathbf{u}, \mathbf{B}), p) \in \mathcal{W}_{\mathbf{g} q}(\Omega) \times L_{0}^{2}(\Omega)$.

Proof. The theorem follows easily from the abstract results in [5] for nonlinear problems and the stated properties of the various forms (for details see [12]).

\section{Finite element approximation}

In this section we present the discrete approximation of problem (3.5). For convenience, we restrict the analysis of the case where $\Omega$ is a convex polyhedral domain. For the approximation we choose finite-dimensional spaces $\mathbf{X}^{h} \subset \mathbf{H}^{1}(\Omega)$, $\mathbf{Y}^{h} \subset \mathbf{H}^{1}(\Omega)$, and $S_{0}^{h} \subset L_{0}^{2}(\Omega)$, and define the following spaces: $\mathbf{X}_{0}^{h}:=\mathbf{X}^{h} \cap \mathbf{H}_{0}^{1}(\Omega)$, $\mathbf{Y}_{n}^{h}:=\mathbf{Y}^{h} \cap \mathbf{H}_{n}^{1}(\Omega), \mathcal{W}^{h}:=\mathbf{X}^{h} \times \mathbf{Y}^{h}$, and $\mathcal{W}_{0 n}^{h}:=\mathbf{X}_{0}^{h} \times \mathbf{Y}_{n}^{h}$. The norms on these 
spaces are induced by the analogous infinite-dimensional spaces. Finally, we define the space of the discrete divergence-free functions, i.e.,

$$
\mathbf{Z}^{h}:=\left\{\mathbf{w}_{h} \in \mathbf{X}_{0}^{h}: \int_{\Omega}\left(\nabla \cdot \mathbf{w}_{h}\right) \chi_{h}=0 \forall \chi_{h} \in S_{0}^{h}\right\} .
$$

Note that, in general, $\mathbf{Z}^{h} \not \subset \mathbf{Z}(\Omega)$. Therefore we must slightly change the nonlinear form $a_{1}$ in order to preserve the useful antisymmetry condition (3.6) also for discrete functions. Thus, we define

$$
\begin{aligned}
\tilde{a}_{1}\left(\left(\mathbf{u}_{h}, \mathbf{B}_{h}\right),\left(\mathbf{v}_{h}, \mathbf{\Psi}_{h}\right),\left(\mathbf{w}_{h}, \mathbf{\Phi}_{h}\right)\right) \\
:=\frac{1}{2} a_{1}\left(\left(\mathbf{u}_{h}, \mathbf{B}_{h}\right),\left(\mathbf{v}_{h} \mathbf{\Psi}_{h}\right),\left(\mathbf{w}_{h}, \mathbf{\Phi}_{h}\right)\right)-\frac{1}{2} a_{1}\left(\left(\mathbf{u}_{h}, \mathbf{B}_{h}\right),\left(\mathbf{w}_{h}, \boldsymbol{\Phi}_{h}\right),\left(\mathbf{v}_{h}, \mathbf{\Psi}_{h}\right)\right) \\
=\frac{M^{2}}{2 N} \int_{\Omega}\left\{\left[\left(\mathbf{u}_{h} \cdot \nabla\right) \mathbf{v}_{h}\right] \cdot \mathbf{w}_{h}-\left[\left(\mathbf{u}_{h} \cdot \nabla\right) \mathbf{w}_{h}\right] \cdot \mathbf{v}_{h}\right\} \\
\quad-R_{m} \int_{\Omega}\left[\left(\nabla \times \mathbf{\Psi}_{h}\right) \times \mathbf{B}_{h}\right] \cdot \mathbf{w}_{h}+R_{m} \int_{\Omega}\left[\left(\nabla \times \mathbf{\Phi}_{h}\right) \times \mathbf{B}_{h}\right] \cdot \mathbf{v}_{h} .
\end{aligned}
$$

It is easy to check that $a_{1}$ and $\tilde{a}_{1}$ are identical for functions $\mathbf{u} \in \mathbf{Z}(\Omega)$. In addition, we now have, by construction,

$$
\tilde{a}_{1}((\mathbf{u}, \mathbf{B}),(\mathbf{v}, \mathbf{\Psi}),(\mathbf{w}, \boldsymbol{\Phi}))=-\tilde{a}_{1}((\mathbf{u}, \mathbf{B}),(\mathbf{w}, \mathbf{\Phi}),(\mathbf{v}, \mathbf{\Psi}))
$$

on all of $\mathcal{W}(\Omega) \times \mathcal{W}(\Omega) \times \mathcal{W}(\Omega)$.

We also modify the form $a$ and define for $\left(\mathbf{u}_{h}, \mathbf{B}_{h}\right),\left(\mathbf{v}_{h}, \mathbf{\Psi}_{h}\right),\left(\mathbf{w}_{h}, \mathbf{\Phi}_{h}\right) \in \mathcal{W}^{h} \times$ $\mathcal{W}^{h} \times \mathcal{W}^{h}$

$$
\begin{aligned}
& \tilde{a}\left(\left(\mathbf{u}_{h}, \mathbf{B}_{h}\right),\left(\mathbf{v}_{h}, \mathbf{\Psi}_{h}\right),\left(\mathbf{w}_{h}, \mathbf{\Phi}_{h}\right)\right) \\
& \quad:=a_{0}\left(\left(\mathbf{v}_{h}, \mathbf{\Psi}_{h}\right),\left(\mathbf{w}_{h}, \mathbf{\Phi}_{h}\right)\right)+\tilde{a}_{1}\left(\left(\mathbf{u}_{h}, \mathbf{B}_{h}\right),\left(\mathbf{v}_{h}, \mathbf{\Psi}_{h}\right),\left(\mathbf{w}_{h}, \mathbf{\Phi}_{h}\right)\right) .
\end{aligned}
$$

For the discrete weak formulation we need to approximate the essential boundary data $\mathbf{g}$ and $q$ by functions $\left.\mathbf{g}_{h} \in \mathbf{X}^{h}\right|_{\Gamma}$ and $q_{h} \in\left\{\left.\left(\boldsymbol{\Psi}_{h} \cdot \mathbf{n}\right)\right|_{\Gamma}: \mathbf{\Psi}_{h} \in \mathbf{Y}^{h}\right\}$. We then have the discrete problem (with $\mathcal{W}_{\mathbf{g}_{h} q_{h}}$ similar to $\mathcal{W}_{\mathbf{g} q}(\Omega)$ in Section 2):

$$
\left\{\begin{array}{c}
\text { Find }\left(\mathbf{u}_{h}, \mathbf{B}_{h}\right) \in \mathcal{W}_{\mathbf{g}_{h} q_{h}} \text { and } p_{h} \in S_{0}^{h} \text { such that } \\
\tilde{a}\left(\left(\mathbf{u}_{h}, \mathbf{B}_{h}\right),\left(\mathbf{u}_{h}, \mathbf{B}_{h}\right),\left(\mathbf{v}_{h}, \mathbf{\Psi}_{h}\right)\right)+b\left(\left(\mathbf{v}_{h}, \mathbf{\Psi}_{h}\right), p_{h}\right)=F_{h}\left(\left(\mathbf{v}_{h}, \mathbf{\Psi}_{h}\right)\right) \forall\left(\mathbf{v}_{h}, \mathbf{\Psi}_{h}\right) \in \mathcal{W}_{0 n}^{h}, \\
b\left(\left(\mathbf{u}_{h}, \mathbf{B}_{h}\right), \chi_{h}\right)=0 \quad \forall \chi_{h} \in S_{0}^{h} .
\end{array}\right.
$$

with

$$
F_{h}\left(\left(\mathbf{v}_{h}, \mathbf{\Psi}_{h}\right)\right)=M^{2} \int_{\Omega} \mathbf{f} \cdot \mathbf{v}_{h}+\left.M \int_{\Gamma} \mathbf{k} \cdot \mathbf{\Psi}_{h}\right|_{\Gamma} .
$$

Remark 5.1. For the discrete spaces $\mathbf{X}^{h}, \mathbf{Y}^{h}$, and $S_{0}^{h}$ we choose finite element spaces. In order to obtain a stable approximation, we have to guarantee that the spaces $\mathbf{X}^{h}$ and $S_{0}^{h}$ satisfy the discrete inf-sup condition

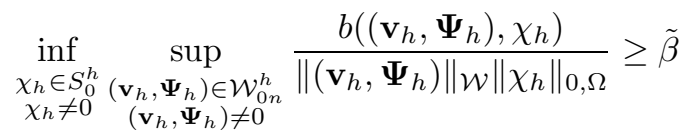

with $\tilde{\beta}>0$ and independent of $h$. 
Remark 5.2. In order to obtain error estimate (see Section 6) we have to make additional regularity assumptions on the boundary data $\mathbf{g}$ and $q$, namely

$$
\mathbf{g} \in \mathbf{H}^{3 / 2+\varepsilon}(\Omega), \quad q \in H^{3 / 2+\varepsilon}(\Omega) .
$$

As a consequence, $\mathbf{g}$ and $q$ are continuous and we can choose for the approximation $q_{h}$ of $q$ the interpolant of $q$ at the boundary vertices. In order to get an existence result we have to construct the discrete function $\mathbf{g}_{h}$ so that it satisfies the compability condition $\int_{\Gamma} \mathbf{g}_{h} \cdot \mathbf{n}=0$. This leads to a construction which we will not present in detail, but which can be found in [6] and also in [12]. We note that this construction is only of theoretical interest, and can be neglected in many practical computations [8].

Next we will derive an existence result for the discrete problem (5.4). For this purpose we closely mimic Section 4 by constructing suitable continuations of the discrete boundary functions $\mathbf{g}_{h}$ and $q_{h}$. We begin by stating a result from [9] without proof.

Lemma 5.3. For any $\varepsilon>0$ there is a real number $h_{0}:=h_{0}(\varepsilon)>0$ such that for all $h$ with $0<h \leq h_{0}$ and for all $q_{h} \in\left\{\left.\left(\boldsymbol{\Psi}_{h} \cdot \mathbf{n}\right)\right|_{\Gamma}: \mathbf{\Psi}_{h} \in \mathbf{Y}^{h}\right\}$ there is a function $\mathbf{B}_{h, 0} \in \mathbf{Y}^{h}$ with

$$
\mathbf{B}_{h, 0} \cdot \mathbf{n}=q_{h} \quad \text { on } \Gamma
$$

and

$$
\left\|\nabla \times \mathbf{B}_{h, 0}\right\|_{0, \Omega} \leq \varepsilon \quad \text { and } \quad\left\|\nabla \cdot \mathbf{B}_{h, 0}\right\|_{0, \Omega} \leq \varepsilon .
$$

Moreover, there is a $\tilde{\gamma}_{2}>0$ such that

$$
\left\|\mathbf{B}_{h, 0}\right\|_{1, \Omega} \leq \tilde{\gamma}_{2}\left\|q_{h}\right\|_{1 / 2, \Gamma} .
$$

For the construction of a discrete continuation of $\mathbf{g}_{h}$ we first define $\mathbf{u}_{\mathbf{g}_{h}} \in \mathbf{H}^{1}(\Omega)$ as the continuous continuation of the discrete boundary data $\mathbf{g}_{h}$ with the following properties (see Lemma 4.3):

$$
\mathbf{u}_{\mathbf{g}_{h}}=\mathbf{g}_{h} \quad \text { on } \Gamma, \quad \nabla \cdot \mathbf{u}_{\mathbf{g}_{h}}=0 \quad \text { in } \Omega,
$$

and for a given $\eta_{1}>0$ :

$$
\left\|\mathbf{u}_{\mathbf{g}_{h}}\right\|_{\mathbf{L}^{3}(\Omega)} \leq \eta_{1}\left\|\mathbf{g}_{h}\right\|_{1 / 2, \Gamma} \quad \text { and } \quad\left\|\mathbf{u}_{\mathbf{g}_{h}}\right\|_{1, \Omega} \leq c\left\|\mathbf{g}_{h}\right\|_{1 / 2, \Gamma} .
$$

In addition, it follows from the proof of Lemma 4.3 that $\mathbf{u}_{\mathbf{g}_{h}}$ has a small support in $\Omega$.

To keep the exposition simpler we restrict ourselves for the moment to scalar functions. We also need some additional notation. Let $\mathcal{N}_{h}$ be the set of all vertices, $\mathcal{N}_{h, \Omega}$ the set of all interior vertices, and $\mathcal{N}_{h, \Gamma}$ the set of all vertices on the boundary $\Gamma$ of the finite element discretization. The vertices are denoted by $x_{i}$, and the nodal basis functions belonging to each vertex by $\mu_{i}$. Then we define $P_{i}$ as the local $L^{2}$-projection onto the support $S_{i}$ of $\mu_{i}$, i.e.,

$$
\begin{aligned}
P_{i}: L^{2}\left(S_{i}\right) & \rightarrow \Pi_{k}\left(S_{i}\right), \\
u & \mapsto P_{i} u \quad \text { with } \int_{S_{i}}\left(P_{i} u-u\right) \pi=0 \forall \pi \in \Pi_{k}\left(S_{i}\right) .
\end{aligned}
$$


Here $\Pi_{k}\left(S_{i}\right)$ denotes the space of polynomials of degree $k$ on $S_{i}$. Our projection operator is a slightly modified interpolation operator of Clément's type [4]:

$$
\begin{aligned}
I_{h}: L^{2}(\Omega) & \rightarrow X^{h} \\
u & \mapsto I_{h} u:=\sum_{x_{i} \in \mathcal{N}_{h, \Omega}}\left(P_{i} u\right)\left(x_{i}\right) \mu_{i}+\sum_{x_{i} \in \mathcal{N}_{h, \Gamma}} g_{h}\left(x_{i}\right) \mu_{i} .
\end{aligned}
$$

On the one hand, this operator preserves the approximation properties of the standard Clément operator, and on the other hand the condition $u_{g_{h}}=g_{h}$ on $\Gamma$, i.e., $I_{h} u_{g_{h}}=g_{h}$ on $\Gamma$.

Lemma 5.4. There exists a constant $c>0$ which is independent on $h$ such that for $u_{g_{h}} \in H^{m}(\Omega)$ the following interpolation estimate holds:

$$
\left|I_{h} u_{g_{h}}-u_{g_{h}}\right|_{k, \Omega} \leq\left.\left. c h^{m-k}\right|_{g_{h}}\right|_{m, \Omega}, \quad 0 \leq k \leq m .
$$

Proof. Denote by $\Pi: L^{2}(\Omega) \rightarrow X^{h}$ the usual Clément interpolation operator (see [4]). Then we have

$$
\begin{aligned}
\left|I_{h} u_{g_{h}}-u_{g_{h}}\right|_{k, \Omega} & \leq\left|I_{h} u_{g_{h}}-\Pi u_{g_{h}}\right|_{k, \Omega}+\left|\Pi u_{g_{h}}-u_{g_{h}}\right|_{k, \Omega} \\
& \leq \underbrace{\left|I_{h} u_{g_{h}}-\Pi u_{g_{h}}\right|_{k, \Omega}}_{=(1)}+c_{1} h^{m-k}\left|u_{g_{h}}\right|_{m, \Omega} .
\end{aligned}
$$

For the estimate of the term (1) in (5.11) we need the following result for an arbitrary boundary simplex $T \in \mathcal{T}_{h, \Gamma}$ :

$$
\left|I_{h} u_{g_{h}}-\Pi u_{g_{h}}\right|_{k, T}^{2} \leq c_{2} h_{T}^{2(m-k)} \sum_{x_{i} \in \mathcal{N}_{h, \Gamma} \cap T}\left|u_{g_{h}}\right|_{m, S_{i}}^{2}
$$

which can be established as follows. From the construction of $I_{h}$ we have

$$
\begin{aligned}
I_{h} u_{g_{h}}-\Pi u_{g_{h}} & =\sum_{x_{i} \in \mathcal{N}_{h, \Omega}}\left(P_{i} u_{g_{h}}\right)\left(x_{i}\right) \mu_{i}+\sum_{x_{i} \in \mathcal{N}_{h, \Gamma}} g_{h}\left(x_{i}\right) \mu_{i}-\sum_{x_{i} \in \mathcal{N}_{h}}\left(P_{i} u_{g_{h}}\right)\left(x_{i}\right) \mu_{i} \\
& =\sum_{x_{i} \in \mathcal{N}_{h, \Gamma}}\left\{g_{h}\left(x_{i}\right)-\left(P_{i} u_{g_{h}}\right)\left(x_{i}\right)\right\} \mu_{i} .
\end{aligned}
$$

Thus for an arbitrary $T \in \mathcal{T}_{h, \Gamma}$ we obtain

$$
\begin{aligned}
\left|I_{h} u_{g_{h}}-\Pi u_{g_{h}}\right|_{k, T}=\left|\sum_{x_{i} \in \mathcal{N}_{h, \Gamma} \cap T}\left\{g_{h}\left(x_{i}\right)-\left(P_{i} u_{g_{h}}\right)\left(x_{i}\right)\right\} \mu_{i}\right|_{k, T} \\
\leq \sum_{x_{i} \in \mathcal{N}_{h, \Gamma} \cap T}|\underbrace{\left\{g_{h}\left(x_{i}\right)-u_{g_{h}}\left(x_{i}\right)\right\}}_{=0} \mu_{i}|_{k, T} \\
\quad+\sum_{x_{i} \in \mathcal{N}_{h, \Gamma}}\left|\left\{\left(P_{i} u_{g_{h}}\right)\left(x_{i}\right)-u_{g_{h}}\left(x_{i}\right)\right\} \mu_{i}\right|_{k, T} \\
\leq c_{3} h_{T}^{-k}\{\operatorname{meas}(T)\}^{1 / 2} \sum_{x_{i} \in \mathcal{N}_{h, \Gamma} \cap T}\left|\left(P_{i} u_{g_{h}}\right)\left(x_{i}\right)-u_{g_{h}}\left(x_{i}\right)\right| .
\end{aligned}
$$


For the term $\left|\left(P_{i} u_{g_{h}}-u_{g_{h}}\right)\left(x_{i}\right)\right|$ the following estimate holds (for details see [12]):

$$
\begin{aligned}
& \left|\left(P_{i} u_{g_{h}}\right)\left(x_{i}\right)-u_{g_{h}}\left(x_{i}\right)\right| \leq\left\|\left.\left(P_{i} u_{g_{h}}-u_{g_{h}}\right)\right|_{\tau}\right\|_{L^{\infty}(\tau)} \\
& \quad \leq c_{6} h_{T}^{-3 / 2}\left\{\left\|P_{i} u_{g_{h}}-u_{g_{h}}\right\|_{0, T}^{2}+h_{T}^{2}\left|P_{i} u_{g_{h}}-u_{g_{h}}\right|_{1, T}^{2}\right\}^{1 / 2} .
\end{aligned}
$$

Here, $x_{i}$ is an arbitrary vertex of a boundary edge $\tau \subset T \in \mathcal{T}_{h, \Gamma}$.

From (5.13) and (5.14) we obtain with the regularity assumption for finite elements (see [3]) and properties of the $L^{2}$-projection (see [4]):

$$
\begin{aligned}
& \left|I_{h} u_{g_{h}}-\Pi u_{g_{h}}\right| k, T \leq c_{7} h_{T}^{3 / 2} h_{T}^{-k} \sum_{x_{i} \in \mathcal{N}_{h, \Gamma} \cap T}\left|\left(P_{i} u_{g_{h}}\right)\left(x_{i}\right)-u_{g_{h}}\left(x_{i}\right)\right| \\
& \leq c_{8} h_{T}^{3 / 2} h_{T}^{-k} \sum_{x_{i} \in \mathcal{N}_{h, \Gamma} \cap T} h_{T}^{-3 / 2}\left\{\left\|P_{i} u_{g_{h}}-u_{g_{h}}\right\|_{0, T}^{2}+h_{T}^{2}\left|P_{i} u_{g_{h}}-u_{g_{h}}\right|_{1, T}^{2}\right\}^{1 / 2} \\
& \quad \leq c_{9} h_{T}^{m-k}\left\{\sum_{x_{i} \in \mathcal{N}_{h, \Gamma} \cap T}\left|u_{g_{h}}\right|_{m, S_{i}}^{2}\right\}^{1 / 2} .
\end{aligned}
$$

Then (5.12) follows easily with $c_{2}=c_{9}^{2}$.

With (5.12) we further obtain

$$
\begin{aligned}
\left|I_{h} u_{g_{h}}-\Pi u_{g_{h}}\right|_{k, \Omega}^{2} & =\sum_{T \in \mathcal{T}_{h}}\left|I_{h} u_{g_{h}}-\Pi u_{g_{h}}\right|_{k, T}^{2} \\
& =\sum_{T \in \mathcal{T}_{h, \Gamma}}\left|I_{h} u_{g_{h}}-\Pi u_{g_{h}}\right|_{k, T}^{2} \\
& \leq c_{10} \sum_{x_{i} \in \mathcal{N}_{h, \Gamma}} h_{T}^{2(m-k)}\left|u_{g_{h}}\right|_{m, S_{i}}^{2} \\
& \leq c_{11} h^{2(m-k)} \sum_{x_{i} \in \mathcal{N}_{h, \Gamma}}\left|u_{g_{h}}\right|_{m, T_{i}}^{2} \\
& \leq c_{12} h^{2(m-k)} \sum_{T \in \mathcal{T}_{h, \Gamma}}\left|u_{g_{h}}\right|_{m, T}^{2} \\
& =c_{12} h^{2(m-k)}\left|u_{g_{h}}\right|_{m, \Omega}^{2} .
\end{aligned}
$$

Finally, (5.11) and (5.15) yield the claimed estimate.

From Lemma 5.4 we have the following corollary, which we present without proof (a proof can be found in [12]).

Corollary 5.5. Denote by $u_{g_{h}} \in H^{1}(\Omega)$ the continuation of $\left.g_{h} \in X^{h}\right|_{\Gamma}$ as in (5.8) and by $I_{h}$ the projection operator defined in (5.10). Then for every $n_{1}>0$ there exist two constants $c_{1}, c_{2}>0$, both independent of $h$, such that

(1) $\left\|I_{h} u_{g_{h}}\right\|_{1, \Omega} \leq c_{1}\left\|g_{h}\right\|_{1 / 2, \Gamma}$,

(2) $\left\|I_{h} u_{g_{h}}\right\|_{L^{3}(\Omega)} \leq c_{2}\left(\eta_{1}+h^{1 / 2}\right)\left\|g_{h}\right\|_{1 / 2, \Gamma}$. 
We define $\mathbf{u}_{h, \mathbf{g}_{h}}:=I_{h} \mathbf{u}_{\mathbf{g}_{h}}$. This function has the following properties $\left(\eta_{1}>0\right.$, $c_{1}, c_{2}$ two constants independent of $h$ ):

$$
\left\{\begin{array}{l}
\text { (1) } \mathbf{u}_{h, \mathbf{g}_{h}}=\mathbf{g}_{h} \text { on } \Gamma \\
\text { (2) } \int_{\Gamma} \mathbf{u}_{h, \mathbf{g}_{h}} \cdot \mathbf{n}=0 \\
\text { (3) }\left\|\mathbf{u}_{h, \mathbf{g}_{h}}\right\|_{1, \Omega} \leq c_{1}\left\|\mathbf{g}_{h}\right\|_{1 / 2, \Gamma} \\
\text { (4) }\left\|\mathbf{u}_{h, \mathbf{g}_{h}}\right\|_{\mathbf{L}^{3}(\Omega)} \leq c_{2}\left(\eta_{1}+h^{1 / 2}\right)\left\|\mathbf{g}_{h}\right\|_{1 / 2, \Gamma} \\
\text { (5) } \mathbf{u}_{h, \mathbf{g}_{h}} \text { has a small support in } \Omega .
\end{array}\right.
$$

Finally we construct from $\mathbf{u}_{h, \mathbf{g}_{h}}$ with a Stokes-projection a solenoidal continuation of the discrete boundary function $\mathbf{g}_{h}$.

Lemma 5.6. For all $\varepsilon>0$ there is an $h_{0}:=h_{0}(\varepsilon)>0$ such that for all $0<h \leq h_{0}$ and all $\left.\mathbf{g}_{h} \in \mathbf{X}^{h}\right|_{\Gamma}$ as in Remark 5.2 there is a function $\mathbf{u}_{h, 0} \in \mathbf{X}^{h}$ with the following properties $\left(c_{1}>0\right.$ a constant independent of $\left.h\right)$ :

(1) $\int_{\Omega}\left(\nabla \cdot \mathbf{u}_{h, 0}\right) \chi_{h}=0 \forall \chi_{h} \in S_{0}^{h}$.

(2) $\mathbf{u}_{h, 0}=\mathbf{g}_{h}$ on $\Gamma$.

(3) $\left\|\mathbf{u}_{h, 0}\right\|_{1, \Omega} \leq c_{1}\left\|\mathbf{g}_{h}\right\|_{1 / 2, \Gamma}$.

(4) $\left\|\mathbf{u}_{h, 0}\right\|_{\mathbf{L}^{3}(\Omega)} \leq \varepsilon\left\|\mathbf{g}_{h}\right\|_{1 / 2, \Gamma}$.

Proof. Let $\mathbf{u}_{h, \mathbf{g}_{h}} \in \mathbf{X}^{h}$ be the continuation of $\mathbf{g}_{h}$ as in (5.16). We denote by $\hat{\mathbf{u}}_{h} \in \mathbf{X}_{0}^{h}, \hat{p}_{h} \in S_{0}^{h}$ the solution of the following well-posed Stokes problem:

$$
\left\{\begin{aligned}
\text { Find } \hat{\mathbf{u}}_{h} \in \mathbf{X}_{0}^{h} \text { and } \hat{p}_{h} \in S_{0}^{h} \text { such that } & \\
\left(\nabla \hat{\mathbf{u}}_{h}, \nabla \mathbf{v}_{h}\right)_{L^{2}}-\left(\nabla \cdot \mathbf{v}_{h}, \hat{p}_{h}\right)_{L^{2}}=\left(\nabla \mathbf{u}_{h, \mathbf{g}_{h}}, \nabla \mathbf{v}_{h}\right)_{L^{2}} & \forall \mathbf{v}_{h} \in \mathbf{X}_{0}^{h}, \\
\left(\nabla \cdot \hat{\mathbf{u}}_{h}, \chi_{h}\right)_{L^{2}}=\left(\nabla \cdot \mathbf{u}_{h, \mathbf{g}_{h}}, \chi_{h}\right)_{L^{2}} & \forall \chi_{h} \in S_{0}^{h} .
\end{aligned}\right.
$$

Here $(\cdot, \cdot)_{L^{2}}$ denotes the usual $L^{2}$-scalar product. Define $\mathbf{u}_{h, 0}:=\mathbf{u}_{h, \mathbf{g}_{h}}-\hat{\mathbf{u}}_{h}$. It follows from (5.17) that $\mathbf{u}_{h, 0}$ is discretely solenoidal and, in addition,

$$
\left.\mathbf{u}_{h, 0}\right|_{\Gamma}=\left.\mathbf{u}_{h, \mathbf{g}_{h}}\right|_{\Gamma}=\mathbf{g}_{h} .
$$

It remains to prove (3) and (4). From an a priori estimate for the Stokes equations we know that (see [5])

$$
\begin{aligned}
\left\|\hat{\mathbf{u}}_{h}\right\|_{1, \Omega}+\left\|\hat{p}_{h}\right\|_{0, \Omega} & \leq c_{2}\left\{\left\|\nabla \mathbf{u}_{h, \mathbf{g}_{h}}\right\|_{0, \Omega}+\left\|\nabla \cdot \mathbf{u}_{h, \mathbf{g}_{h}}\right\|_{0, \Omega}\right\} \\
& \leq c_{3}\left\|\mathbf{u}_{h, \mathbf{g}_{h}}\right\|_{1, \Omega} .
\end{aligned}
$$

This immediately yields

$$
\left\|\hat{\mathbf{u}}_{h}\right\|_{1, \Omega} \leq c_{3}\left\|\mathbf{u}_{h, \mathbf{g}_{h}}\right\|_{1, \Omega} .
$$

Together with (5.16(3)) and (5.19) this yields (3):

$$
\left\|\mathbf{u}_{h, 0}\right\|_{1, \Omega}=\left\|\mathbf{u}_{h, \mathbf{g}_{h}}-\hat{\mathbf{u}}_{h}\right\|_{1, \Omega} \leq\left\|\mathbf{u}_{h, \mathbf{g}_{h}}\right\|_{1, \Omega}+\left\|\hat{\mathbf{u}}_{h}\right\|_{1, \Omega} \leq c_{1}\left\|\mathbf{g}_{h}\right\|_{1 / 2, \Gamma}
$$

For the $\mathbf{L}^{3}$-estimate of $\mathbf{u}_{h, 0}$ we need an estimate in the $\mathbf{L}^{2}$-norm for $\hat{\mathbf{u}}_{h}$. For this purpose, we solve the following problem:

$$
\left\{\begin{aligned}
\text { Find } \mathbf{u} \in \mathbf{H}_{0}^{1}(\Omega) \text { and } p \in L_{0}^{2}(\Omega) \text { such that } & \\
(\nabla \mathbf{u}, \nabla \mathbf{v})_{L^{2}}+(\nabla \cdot \mathbf{v}, p)_{L^{2}}=\left(\nabla \hat{\mathbf{u}}_{h}, \mathbf{v}\right)_{L^{2}} & \forall \mathbf{v} \in \mathbf{H}_{0}^{1}(\Omega), \\
-(\nabla \cdot \mathbf{u}, \chi)_{L^{2}}=0 & \forall \chi \in L_{0}^{2}(\Omega) .
\end{aligned}\right.
$$


Therefore for arbitrary $\mathbf{v}_{h} \in \mathbf{X}^{h}, \chi_{h} \in S_{0}^{h}$ with $\mathbf{v}:=\hat{\mathbf{u}}_{h}, \chi:=\hat{p}_{h}$ we obtain

$$
\begin{aligned}
\left\|\hat{\mathbf{u}}_{h}\right\|_{0, \Omega}^{2}=( & \left.\left(\mathbf{u}-\mathbf{v}_{h}\right), \nabla \hat{\mathbf{u}}_{h}\right)_{L^{2}}-\left(\nabla \cdot\left(\mathbf{u}-\mathbf{v}_{h}\right), \hat{p}_{h}\right)_{L^{2}}+\left(\nabla \cdot \hat{\mathbf{u}}_{h},\left(p-\chi_{h}\right)\right)_{L^{2}} \\
& +\left(\nabla \hat{\mathbf{u}}_{h}, \nabla \mathbf{v}_{h}\right)_{L^{2}}-\left(\nabla \cdot \mathbf{v}_{h}, \hat{p}_{h}\right)_{L^{2}}+\left(\nabla \cdot \hat{\mathbf{u}}_{h}, \chi_{h}\right)_{L^{2}} \\
=( & \left.\left(\mathbf{u}-\mathbf{v}_{h}\right), \nabla \hat{\mathbf{u}}_{h}\right)_{L^{2}}-\left(\nabla \cdot\left(\mathbf{u}-\mathbf{v}_{h}\right), \hat{p}_{h}\right)_{L^{2}}+\left(\nabla \cdot \hat{\mathbf{u}}_{h},\left(p-\chi_{h}\right)\right)_{L^{2}} \\
& +\left(\nabla \mathbf{u}_{h, \mathbf{g}_{h}}, \nabla\left(\mathbf{v}_{h}-\mathbf{u}\right)\right)_{L^{2}}+\left(\nabla \cdot \mathbf{u}_{h, \mathbf{g}_{h}},\left(\chi_{h}-p\right)\right)_{L^{2}} \\
& +\left(\nabla \mathbf{u}_{h, \mathbf{g}_{h}}, \nabla \mathbf{u}\right)_{L^{2}}+\left(\nabla \cdot \mathbf{u}_{h, \mathbf{g}_{h}}, p\right)_{L^{2}} \\
\leq\{ & \left\|\mathbf{u}-\mathbf{v}_{h}\right\|_{1, \Omega}\left\|\hat{\mathbf{u}}_{h}\right\|_{1, \Omega}+\left\|\mathbf{u}-\mathbf{v}_{h}\right\|_{1, \Omega}\left\|\hat{p}_{h}\right\|_{0, \Omega}+\left\|\hat{\mathbf{u}}_{h}\right\|_{1, \Omega}\left\|p-\chi_{h}\right\|_{0, \Omega} \\
\left.+\left\|\mathbf{u}_{h, \mathbf{g}_{h}}\right\|_{1, \Omega}\left\|\mathbf{v}_{h}-\mathbf{u}\right\|_{1, \Omega}+\left\|\mathbf{u}_{h, \mathbf{g}_{h}}\right\|_{1, \Omega}\left\|\chi_{h}-p\right\|_{0, \Omega}\right\} & \\
& +\left(\nabla \mathbf{u}_{h, \mathbf{g}_{h}}, \nabla \mathbf{u}\right)_{L^{2}}+\left(\nabla \cdot \mathbf{u}_{h, \mathbf{g}_{h}}, p\right)_{L^{2}} \\
\leq & c_{4} h\left\{\left\|\hat{\mathbf{u}}_{h}\right\|_{1, \Omega}+\left\|\hat{p}_{h}\right\|_{0, \Omega}+\left\|\mathbf{u}_{h, \mathbf{g}_{h}}\right\|_{1, \Omega}\right\}\left\{\|\mathbf{u}\|_{2, \Omega}+\|p\|_{1, \Omega}\right\} \\
& +\underbrace{\left(\nabla \mathbf{u}_{h, \mathbf{g}_{h}}, \nabla \mathbf{u}\right)_{L^{2}}+\left(\nabla \cdot \mathbf{u}_{h, \mathbf{g}_{h}}, p\right)_{L^{2}}}_{=(1)} .
\end{aligned}
$$

Define $\underline{\underline{\mathbf{T}}}:=\nabla \mathbf{u}+p \underline{\underline{\mathbf{I}}}$. Because of $(5.16(5))$ there exists a real $\eta_{2}>0$ with

$$
\operatorname{supp}\left(\mathbf{u}_{h, \mathbf{g}_{h}}\right) \subset \Gamma_{\eta_{2}}:=\left\{x \in \Omega: d(x, \Gamma) \leq \eta_{2}\right\}
$$

We then obtain

$$
\begin{aligned}
(1) & =\left(\nabla \mathbf{u}_{h, \mathbf{g}_{h}}, \underline{\underline{\mathbf{T}}}\right)_{L^{2}}=\int_{\Omega}\left(\nabla \mathbf{u}_{h, \mathbf{g}_{h}} \cdot \underline{\underline{\mathbf{T}}}\right) \chi_{\Gamma_{\eta_{2}}} \\
& \leq c_{5}\left\|\nabla \mathbf{u}_{h, \mathbf{g}_{h}}\right\|_{0, \Omega}\|\underline{\underline{\mathbf{T}}}\|_{\mathbf{L}^{6}(\Omega)}\left\|\chi_{\Gamma_{\eta_{2}}}\right\|_{\mathbf{L}^{3}(\Omega)} \\
& \leq c_{6}\left\|\mathbf{u}_{h, \mathbf{g}_{h}}\right\|_{1, \Omega}\left\{\|\mathbf{u}\|_{2, \Omega}+\|p\|_{1, \Omega}\right\} \eta_{2}^{1 / 3},
\end{aligned}
$$

where we have used a generalized Hölder inequality and the Sobolev imbedding theorem $\mathbf{H}^{1}(\Omega) \hookrightarrow \mathbf{L}^{6}(\Omega)$. Then the $\mathbf{H}^{2}$-regularity of problem (5.20) and estimates (5.21), (5.22), and (5.18) yield

$$
\left\|\hat{\mathbf{u}}_{h}\right\|_{0, \Omega} \leq c_{7} \frac{\left\|\hat{\mathbf{u}}_{h}\right\|_{0, \Omega}^{2}}{\|\mathbf{u}\|_{2, \Omega}+\|p\|_{1, \Omega}} \leq c_{8}\left(h+\eta_{2}^{1 / 3}\right)\left\|\mathbf{u}_{h, \mathbf{g}_{h}}\right\|_{1, \Omega} .
$$

Finally, (5.19), (5.23), and (5.16) imply

$$
\begin{aligned}
\left\|\mathbf{u}_{h, 0}\right\|_{\mathbf{L}^{3}(\Omega)} & \leq\left\|\hat{\mathbf{u}}_{h}\right\|_{\mathbf{L}^{3}(\Omega)}+\left\|\mathbf{u}_{h, \mathbf{g}_{h}}\right\|_{\mathbf{L}^{3}(\Omega)} \\
& \leq c_{9}\left\|\hat{\mathbf{u}}_{h}\right\|_{0, \Omega}^{1 / 2}\left\|\hat{\mathbf{u}}_{h}\right\|_{\mathbf{L}^{4}(\Omega)}^{1 / 2}+\left\|\mathbf{u}_{h, \mathbf{g}_{h}}\right\|_{\mathbf{L}^{3}(\Omega)} \\
& \leq c_{10}\left\|\hat{\mathbf{u}}_{h}\right\|_{0, \Omega}^{1 / 2}\left\|\hat{\mathbf{u}}_{h}\right\|_{1, \Omega}^{1 / 2}+\left\|\mathbf{u}_{h, \mathbf{g}_{h}}\right\|_{\mathbf{L}^{3}(\Omega)} \\
& \leq c_{11}\left\{\left(h+\eta_{2}^{1 / 3}\right)^{1 / 2}+\left(h^{1 / 2}+\eta_{1}\right)\right\}\left\|\mathbf{g}_{h}\right\|_{1 / 2, \Gamma} .
\end{aligned}
$$

Thus we may for arbitrary $\varepsilon>0$ choose $\eta_{1}, \eta_{2}$ and $h_{0}>0$ with

$$
c_{11}\left\{\left(h_{0}+\eta_{2}^{1 / 3}\right)^{1 / 2}+\left(h_{0}^{1 / 2}+\eta_{1}\right)\right\} \leq \varepsilon .
$$

This yields the estimate (4) for every $0<h \leq h_{0}$.

In order to obtain an existence result for the approximate problem (5.4) we continue as in Section 4. With the continuations of the discrete boundary data $\mathbf{g}_{h}$ and $q_{h}$ we formulate a discrete homogeneous problem analogous to problem (3.7) and then have to show the analogous properties (for example, the coercivity of $\tilde{a}_{1}$ ) as in the continuous case. We refer to [12] for a detailed presentation of this process, 
and state only the main existence result. Note that for the approximate problem, we also have existence of solutions without any conditions on the boundary data.

Theorem 5.7. The approximate problem (5.4) has for every sufficiently small $h>0$ and for every function $\mathbf{f} \in \mathbf{H}^{-1}(\Omega)$, for every boundary function $\mathbf{k}$ with (3.4) and for all discrete boundary functions $\left.\mathbf{g}_{h} \in \mathbf{X}^{h}\right|_{\Gamma}$ with $\int_{\Omega} \mathbf{g}_{h} \cdot \mathbf{n}=0$ and $q_{h} \in\left\{\left.\left(\mathbf{\Psi}_{h} \cdot \mathbf{n}\right)\right|_{\Gamma}: \mathbf{\Psi}_{h} \in \mathbf{Y}^{h}\right\}$ at least one solution $\left(\left(\mathbf{u}_{h}, \mathbf{B}_{h}\right), p\right) \in \mathcal{W}_{\mathbf{g}_{h} q_{h}}^{h} \times S_{0}^{h}$.

We finish this section with an error estimate for the linear case which will be needed for the error estimate in the general nonlinear case in the next section.

Let us formulate the linear problems

$$
\left\{\begin{array}{rlrl}
\text { Find }(\mathbf{u}, \mathbf{B}) \in \mathcal{W}_{\mathbf{g} q}(\Omega) \text { and } p \in L_{0}^{2}(\Omega) \text { such that } & \\
a_{0}((\mathbf{u}, \mathbf{B}),(\mathbf{v}, \mathbf{\Psi}))+b((\mathbf{v}, \mathbf{\Psi}), p)=F((\mathbf{v}, \mathbf{\Psi})) & & \forall(\mathbf{v}, \mathbf{\Psi}) \in \mathcal{W}_{0 n}(\Omega), \\
b((\mathbf{u}, \mathbf{B}), \chi)=0 & & \forall \chi \in L_{0}^{2}(\Omega),
\end{array}\right.
$$

and

$$
\left\{\begin{array}{cl}
\text { Find }\left(\mathbf{u}_{h}, \mathbf{B}_{h}\right) \in \mathcal{W}_{\mathbf{g}_{h} q_{h}}^{h} \text { and } p_{h} \in S_{0}^{h} \text { such that } & \\
a_{0}\left(\left(\mathbf{u}_{h}, \mathbf{B}_{h}\right),\left(\mathbf{v}_{h}, \mathbf{\Psi}_{h}\right)\right)+b\left(\left(\mathbf{v}_{h}, \mathbf{\Psi}_{h}\right), p_{h}\right)=F\left(\left(\mathbf{v}_{h}, \mathbf{\Psi}_{h}\right)\right) & \forall\left(\mathbf{v}_{h}, \mathbf{\Psi}_{h}\right) \in \mathcal{W}_{0 n}^{h} \\
b\left(\left(\mathbf{u}_{h}, \mathbf{B}_{h}\right), \chi_{h}\right)=0 & \forall \chi_{h} \in S_{0}^{h}
\end{array}\right.
$$

With the abstract results of [5] it is easy to prove that the linear problems both have a unique solution. We then have the following error estimate (for details and a proof, see [12]).

Proposition 5.8. Let $(\mathbf{u}, \mathbf{B}) \in \mathbf{H}^{2}(\Omega) \times \mathbf{H}^{2}(\Omega), p \in H^{1}(\Omega)$ and $\left(\mathbf{u}_{h}, \mathbf{B}_{h}\right) \in \mathcal{W}_{\mathbf{g}_{h} q_{h}}^{h}$, $p_{h} \in S_{0}^{h}$ be the unique solutions of problems (5.24) and (5.25). Then there is a constant $c>0$, independent of $h$, such that

$$
\left\|(\mathbf{u}, \mathbf{B})-\left(\mathbf{u}_{h}, \mathbf{B}_{h}\right)\right\|_{\mathcal{W}}+\left\|p-p_{h}\right\|_{0, \Omega} \leq \operatorname{ch}\left\{\|(\mathbf{u}, \mathbf{B})\|_{\mathbf{H}^{2}(\Omega) \times \mathbf{H}^{2}(\Omega)}+\|p\|_{1, \Omega}\right\}
$$

Here the degree of polynomials in $\mathbf{X}^{h}$ and $\mathbf{Y}^{h}$ is at least 1 and is equal for both spaces; the degree of polynomials in $S_{0}^{h}$ is at least 0 . In addition we require the inf-sup condition (5.6).

In Section 6 we derive an error estimate in the general nonlinear case based on the error estimate (5.26). We refer to [9] for an error estimate in the case where both the continuous and the discrete nonlinear problems are uniquely solvable.

\section{ERror estimates For the NONLINEAR CASE}

For the derivation of error estimates in the general nonlinear case we follow the analysis of nonlinear problems in [2] and [5]. We deal with operator problems of the type

$$
\left\{\begin{array}{l}
\text { Find } w \in X \text { such that } \\
\quad F(\lambda, w(\lambda)):=w(\lambda)+T G(\lambda, w(\lambda))=0 .
\end{array}\right.
$$

Here $T: Y \rightarrow X$ is a continuous linear operator, $G$ a $C^{2}$-map from $\Lambda \times X$ to $Y, X, Y$ two Banach spaces and $\Lambda \subset \mathbb{R}^{n}$ compact. We further denote by $\{(\lambda, w(\lambda)): \lambda \in \Lambda\}$ a regular branch of solutions of (6.1) if $\lambda \mapsto w(\lambda)$ is a continuous function from $\Lambda$ into $X$ with $F(\lambda, w(\lambda))=0$ and in addition the derivate $D_{w} F(\lambda, w(\lambda))$ is an isomorphism of $X$ into itself for each $\lambda \in \Lambda$. 
We then approximate problem (6.1). For this purpose we choose finite-dimensional subspaces $X^{h}, Y^{h}$ of $X, Y$ and we approximate the operators $T$ and $G$ by $T^{h} \in \mathcal{L}\left(Y^{h}, X^{h}\right)$ and $G^{h} \in C^{2}\left(\Lambda \times X^{h}, Y^{h}\right)$. Thus we obtain the discrete problem:

$$
\left\{\begin{array}{l}
\text { Find } w_{h} \in X^{h} \text { such that } \\
\qquad F^{h}\left(\lambda, w_{h}(\lambda)\right):=w_{h}(\lambda)+T^{h} G^{h}\left(\lambda, w_{h}(\lambda)\right)=0 .
\end{array}\right.
$$

Remark 6.1. Our operator formulation is slightly different from the one in [5], because we approximate not only the operator $T$, but also the nonlinear operator $G$. This is a consequence of the fact that we have approximated the boundary data $\mathbf{g}$ and $q$ by finite element functions $\mathbf{g}_{h}$ and $q_{h}$. An analogous formulation can be found in [11] for an analysis of a streamline-diffusion finite element method for the Navier-Stokes equations.

Let us now define for our problems (3.5) and (5.4) the spaces $X, Y, X^{h}, Y^{h}$ and the operators $T, G, T^{h}, G^{h}$. We start with

$$
X:=\mathcal{W}(\Omega) \times L_{0}^{2}(\Omega), \quad Y:=Y_{1} \times Y_{2},
$$

with

$$
\begin{aligned}
& Y_{1}:=\mathbf{H}^{-1}(\Omega) \times\left(\mathbf{H}_{n}^{1}(\Omega)\right)^{\prime}, \\
& Y_{2}:=\left\{\mathbf{g}^{*} \in \mathbf{H}^{1 / 2}(\Gamma): \int_{\Omega} \mathbf{g}^{*} \cdot \mathbf{n}=0\right\} \times\left\{q^{*} \in H^{1 / 2}(\Gamma): \int_{\Omega} q^{*}=0\right\} .
\end{aligned}
$$

Next, $T$ denotes the continuous linear operator which maps a given $\left(\left(\mathbf{f}_{1}^{*}, \mathbf{f}_{2}^{*}\right)\right.$, $\left.\left(\mathbf{g}^{*}, q^{*}\right)\right) \in Y$ to the solution $\left(\left(\mathbf{u}^{*}, \mathbf{B}^{*}\right), p^{*}\right)=T\left(\left(\left(\mathbf{f}_{1}^{*}, \mathbf{f}_{2}^{*}\right),\left(\mathbf{g}^{*}, q^{*}\right)\right)\right) \in X$ of the following linear problem $\left(F^{*}((\mathbf{v}, \boldsymbol{\Psi})):=\mathbf{f}_{1}^{*}(\mathbf{v})+\mathbf{f}_{2}^{*}(\boldsymbol{\Psi})\right)$ :

$$
\left\{\begin{array}{ccc}
\text { Find }\left(\left(\mathbf{u}^{*}, \mathbf{B}^{*}\right), p^{*}\right) \in X \text { with } \mathbf{u}^{*}=\mathbf{g}^{*} \text { on } \Gamma \text { and } \mathbf{B}^{*} \cdot \mathbf{n}=q^{*} \text { on } \Gamma \text { such that } \\
a_{0}\left(\left(\mathbf{u}^{*}, \mathbf{B}^{*}\right),(\mathbf{v}, \boldsymbol{\Psi})\right)+b\left((\mathbf{v}, \mathbf{\Psi}), p^{*}\right)=F^{*}((\mathbf{v}, \boldsymbol{\Psi})) & \forall(\mathbf{v}, \boldsymbol{\Psi}) \in \mathcal{W}_{0 n}(\Omega), \\
b\left(\left(\mathbf{u}^{*}, \mathbf{B}^{*}\right), \chi\right)=0 & \forall \chi \in L_{0}^{2}(\Omega) .
\end{array}\right.
$$

With the given data $\mathbf{f}, \mathbf{g}, q$ and $\mathbf{k}$ of problem (3.5) we associate a $C^{\infty}$-function $G$ from $\mathbb{R}_{+}^{3} \times X$ into $Y$, i.e.,

$G:\left(\left(M, R_{m}, N\right),((\mathbf{u}, \mathbf{B}), p)\right) \mapsto G\left(\left(M, R_{m}, N\right),((\mathbf{u}, \mathbf{B}), p)\right):=\left(\left(G_{1}, G_{2}\right),\left(G_{3}, G_{4}\right)\right)$

with $\left((\mathbf{v}, \Psi) \in \mathcal{W}_{0 n}(\Omega)\right)$

$$
\begin{cases}G_{1} & :=\frac{M^{2}}{N} \int_{\Omega}[(\mathbf{u} \cdot \nabla) \mathbf{u}] \cdot \mathbf{v}-R_{m} \int_{\Omega}[(\nabla \times \mathbf{B}) \times \mathbf{B}] \cdot \mathbf{v}-M^{2} \int_{\Omega} \mathbf{f} \cdot \mathbf{v} \\ G_{2} & :=R_{m} \int_{\Omega}[(\nabla \times \mathbf{\Psi}) \times \mathbf{B}] \cdot \mathbf{u}-M\left\langle\mathbf{k},\left.\mathbf{\Psi}\right|_{\Gamma}\right\rangle_{\Gamma}, \\ \left(G_{3}, G_{4}\right) & :=(-\mathbf{g},-q) .\end{cases}
$$

By setting

$$
F\left(\left(M, R_{m}, N\right),((\mathbf{u}, \mathbf{B}), p)\right):=((\mathbf{u}, \mathbf{B}), p)+T G\left(\left(M, R_{m}, N\right),((\mathbf{u}, \mathbf{B}), p)\right),
$$

we obtain the following operator problem:

$$
\left\{\begin{aligned}
\text { Find } & ((\mathbf{u}, \mathbf{B}), p) \in X \text { such that } \\
& F\left(\left(M, R_{m}, N\right),((\mathbf{u}, \mathbf{B}), p)\right)=0
\end{aligned}\right.
$$


Remark 6.2. By construction of the operators $T$ and $G$ it is clear that $((\mathbf{u}, \mathbf{B}), p) \in$ $X$ is a solution of problem (6.5) if and only if $(\mathbf{u}, \mathbf{B}) \in \mathcal{W}_{\mathbf{g} q}(\Omega), p \in L_{0}^{2}(\Omega)$ is a solution of problem (3.5) with given data $\mathbf{f}, \mathbf{g}, q$ and $\mathbf{k}$.

Next we have to write our discrete problem (5.4) also as an operator problem. For this purpose we approximate the spaces and the operators $T$ and $G$ (see Remark 6.1). For the discrete spaces we choose

$$
X^{h}:=\mathcal{W}^{h}, \quad Y^{h}:=Y_{1} \times Y_{2}^{h},
$$

with

$$
Y_{2}^{h}:=\left\{\left.\mathbf{g}_{h}^{*} \in \mathbf{X}^{h}\right|_{\Gamma}: \int_{\Omega} \mathbf{g}_{h}^{*} \cdot \mathbf{n}=0\right\} \times\left\{\left.\left(\mathbf{\Psi}_{h} \cdot \mathbf{n}\right)\right|_{\Gamma}: \mathbf{\Psi}_{h} \in \mathbf{Y}^{h}\right\}
$$

Also, we denote by $T^{h}$ an approximation of $T$ which maps a given $\left(\left(\mathbf{f}_{1}^{*}, \mathbf{f}_{2}^{*}\right)\right.$, $\left.\left(\mathbf{g}_{h}^{*}, q_{h}^{*}\right)\right) \in Y^{h}$ with $\left(\left(\mathbf{u}_{h}^{*}, \mathbf{B}_{h}^{*}\right), p_{h}^{*}\right)=T^{h}\left(\left(\left(\mathbf{f}_{1}^{*}, \mathbf{f}_{2}^{*}\right),\left(\mathbf{g}_{h}^{*}, q_{h}^{*}\right)\right)\right) \in X^{h}$ to the solution of the following linear discrete problem $\left(F^{*}\right.$ as in $\left.(6.3)\right)$ :

$$
\left\{\begin{array}{cl}
\text { Find }\left(\left(\mathbf{u}_{h}^{*}, \mathbf{B}_{h}^{*}\right), p_{h}^{*}\right) \in X^{h} \text { with } \mathbf{u}_{h}^{*}=\mathbf{g}_{h}^{*} \text { on } \Gamma \text { and } \mathbf{B}_{h}^{*} \cdot \mathbf{n}=q_{h}^{*} \text { on } \Gamma \text { such that } \\
a_{0}\left(\left(\mathbf{u}_{h}^{*}, \mathbf{B}_{h}^{*}\right),\left(\mathbf{v}_{h}, \mathbf{\Psi}_{h}\right)\right)+b\left(\left(\mathbf{v}_{h}, \mathbf{\Psi}_{h}\right), p_{h}^{*}\right)=F^{*}\left(\left(\mathbf{v}_{h}, \mathbf{\Psi}_{h}\right)\right) & \forall\left(\mathbf{v}_{h}, \mathbf{\Psi}_{h}\right) \in \mathcal{W}_{0 n}^{h}, \\
b\left(\left(\mathbf{u}_{h}^{*}, \mathbf{B}_{h}^{*}\right), \chi_{h}\right)=0 & \forall \chi_{h} \in S_{0}^{h} .
\end{array}\right.
$$

Next we approximate the operator $G$ by $G^{h}: \mathbb{R}_{+}^{3} \times X^{h} \rightarrow Y^{h}$. Compared to the definition of (6.4), we only have to change the last two components by replacing $\mathbf{g}$ by $\mathbf{g}_{h}$ and $q$ by $q_{h}$, i.e.,

$$
\left(G_{1}^{h}, G_{2}^{h}\right):=\left(G_{1}, G_{2}\right), \quad\left(G_{3}^{h}, G_{4}^{h}\right):=\left(-\mathbf{g}_{h},-q_{h}\right) .
$$

Finally, we set

$$
F^{h}\left(\left(M, R_{m}, N\right),\left(\left(\mathbf{u}_{h}, \mathbf{B}_{h}\right), p_{h}\right)\right):=\left(\left(\mathbf{u}_{h}, \mathbf{B}_{h}\right), p_{h}\right)+T^{h} G^{h}\left(\left(M, R_{m}, N\right),\left(\left(\mathbf{u}_{h}, \mathbf{B}_{h}\right), p_{h}\right)\right)
$$

and obtain the discrete operator problem

$$
\left\{\begin{array}{l}
\text { Find }\left(\left(\mathbf{u}_{h}, \mathbf{B}_{h}\right), p_{h}\right) \in X^{h} \text { such that } \\
\quad F^{h}\left(\left(M, R_{m}, N\right),\left(\left(\mathbf{u}_{h}, \mathbf{B}_{h}\right), p_{h}\right)\right)=0 .
\end{array}\right.
$$

In order to derive an error estimate we need some additional notation. Set $\lambda:=\left(M, R_{m}, N\right) \in \Lambda$ with $\Lambda \subset \mathbb{R}_{+}^{3}$ compact. We further assume that $\lambda \mapsto$ $\left(\left(\mathbf{u}_{\lambda}, \mathbf{B}_{\lambda}\right), p_{\lambda}\right)$ is a regular branch of solutions of problem (6.5) which satisfies the regularity condition $\left(\mathbf{u}_{\lambda}, \mathbf{B}_{\lambda}\right) \in \mathbf{H}^{2}(\Omega) \times \mathbf{H}^{2}(\Omega), p_{\lambda} \in H^{1}(\Omega)$. We set

$$
\gamma(\lambda):=\left\|D_{2} F\left(\lambda,\left(\left(\mathbf{u}_{\lambda}, \mathbf{B}_{\lambda}\right), p_{\lambda}\right)\right)^{-1}\right\|_{\mathcal{L}(X, X)}<\infty
$$

and $\gamma^{*}:=\sup _{\gamma \in \Lambda} \gamma(\lambda)$. Here, $D_{2} F$ denotes the derivative of $F$ with respect to $((\mathbf{u}, \mathbf{B}), p)$. We further define

$$
K:=\max \left\{1,\left\|\left(\mathbf{u}_{\lambda}, \mathbf{B}_{\lambda}\right)\right\|_{\mathbf{H}^{2}(\Omega) \times \mathbf{H}^{2}(\Omega)},\left\|p_{\lambda}\right\|_{1, \Omega},\|\mathbf{g}\|_{3 / 2, \Gamma},\|q\|_{3 / 2, \Gamma},\|f\|_{0, \Omega},\|\mathbf{k}\|_{1 / 2, \Gamma}\right\} .
$$

Finally, we assume that $\left(\left(\hat{\mathbf{u}}_{h, \lambda} \widehat{\mathbf{B}}_{h, \lambda}\right), \hat{p}_{h}\right) \in X^{h}$ is a suitable projection (for example with an operator of Clément's type [4]) of $\left(\left(\mathbf{u}_{\lambda}, \mathbf{B}_{\lambda}\right), p_{\lambda}\right) \in X$. With this notation we obtain

Theorem 6.3. Let $\lambda \mapsto\left(\left(\mathbf{u}_{\lambda}, \mathbf{B}_{\lambda}\right), p_{\lambda}\right)$ be a regular branch of solutions of the operator equation (6.5). Then there exists a real $h_{0}>0$ such that for each $0<h \leq$ $h_{0}$ and each $\lambda \in \Lambda$ the discrete operator problem (6.7) allows a unique solution 
$\left(\left(\mathbf{u}_{h, \lambda}, \mathbf{B}_{h, \lambda}\right), p_{h, \lambda}\right) \in X^{h}$ in a neighbourhood of $\left(\left(\hat{\mathbf{u}}_{h, \lambda}, \widehat{\mathbf{B}}_{h, \lambda}\right), \hat{p}_{h, \lambda}\right)$. Furthermore, we have the error estimate

$$
\left\|\left(\mathbf{u}_{h, \lambda}, \mathbf{B}_{h, \lambda}\right)-\left(\mathbf{u}_{\lambda}, \mathbf{B}_{\lambda}\right)\right\|_{\mathcal{W}}+\left\|p_{h, \lambda}-p_{\lambda}\right\|_{0, \Omega} \leq c h
$$

with $c:=c(\Lambda, K)$.

Proof. We set for abbreviation $\underline{\mathbf{u}}_{\lambda}:=\left(\left(\mathbf{u}_{\lambda}, \mathbf{B}_{\lambda}\right), p_{\lambda}\right), \underline{\hat{\mathbf{u}}}_{h, \lambda}:=\left(\left(\hat{\mathbf{u}}_{h, \lambda}, \widehat{\mathbf{B}}_{h, \lambda}\right), \hat{p}_{h, \lambda}\right)$. With properties of the Clément operator, approximation properties of $\mathbf{g}_{h}$, a priori estimates for the discrete problem and the error estimate (5.26) for the linear problem, we have

$$
\begin{aligned}
\| F^{h}(\lambda, & \left.\underline{\hat{\mathbf{u}}}_{h, \lambda}\right)\left\|_{X}=\right\| F^{h}\left(\lambda, \underline{\hat{\mathbf{u}}}_{h, \lambda}\right)-F\left(\lambda, \underline{\mathbf{u}}_{\lambda}\right) \|_{X} \\
\leq & \left\|\underline{\hat{\mathbf{u}}}_{h, \lambda}-\underline{\mathbf{u}}_{\lambda}\right\|_{X}+\left\|T^{h} G^{h}\left(\lambda, \underline{\hat{\mathbf{u}}}_{h, \lambda}\right)-T^{h} G\left(\lambda, \underline{\hat{\mathbf{u}}}_{h, \lambda}\right)\right\|_{X} \\
& +\left\|T^{h} G\left(\lambda, \underline{\hat{\mathbf{u}}}_{h, \lambda}\right)-T^{h} G\left(\lambda, \underline{\mathbf{u}}_{\lambda}\right)\right\|_{X}+\left\|T^{h} G\left(\lambda, \underline{\mathbf{u}}_{\lambda}\right)-T G\left(\lambda, \underline{\mathbf{u}}_{\lambda}\right)\right\|_{X} \\
& \leq c_{1} h K+c_{2} h K+c_{3} h K+c_{4} h K^{2} \\
& \leq c_{5} h K^{2} .
\end{aligned}
$$

Let $\underline{\mathbf{w}}:=((\mathbf{w}, \boldsymbol{\Phi}), \Upsilon) \in X$ with $\|\underline{\mathbf{w}}\|_{X}=1$. We obtain

$$
\begin{aligned}
&\left\|D_{2} F^{h}\left(\lambda, \underline{\hat{\mathbf{u}}}_{h, \lambda}\right) \underline{\mathbf{w}}-D_{2} F\left(\lambda, \underline{\mathbf{u}}_{\lambda}\right) \underline{\mathbf{w}}\right\|_{X} \\
&=\left\|T^{h} D_{2} G^{h}\left(\lambda, \underline{\hat{\mathbf{u}}}_{h, \lambda}\right) \underline{\mathbf{w}}-T D_{2} G\left(\lambda, \underline{\mathbf{u}}_{\lambda}\right) \underline{\mathbf{w}}\right\|_{X} \\
& \leq\left\|T^{h} D_{2} G^{h}\left(\lambda, \underline{\hat{\mathbf{u}}}_{h, \lambda}\right) \underline{\mathbf{w}}-T^{h} D_{2} G\left(\lambda, \underline{\hat{\mathbf{u}}}_{h, \lambda}\right) \underline{\mathbf{w}}\right\|_{X} \\
&+\left\|T^{h} D_{2} G\left(\lambda, \underline{\hat{\mathbf{u}}}_{h, \lambda}\right) \underline{\mathbf{w}}-T D_{2} G\left(\lambda, \underline{\hat{\mathbf{u}}}_{h, \lambda}\right) \underline{\mathbf{w}}\right\|_{X} \\
&+\left\|T D_{2} G\left(\lambda, \underline{\hat{\mathbf{u}}}_{h, \lambda}\right) \underline{\mathbf{w}}-T D_{2} G\left(\lambda, \underline{\mathbf{u}}_{\lambda}\right) \underline{\mathbf{w}}\right\|_{X} \\
& \leq 0+c_{6} h K+c_{7} h K \\
& \leq c_{8} h K,
\end{aligned}
$$

and therefore

$$
\left\|D_{2} F^{h}\left(\lambda, \underline{\hat{\mathbf{u}}}_{h, \lambda}\right)-D_{2} F\left(\lambda, \underline{\mathbf{u}}_{\lambda}\right)\right\|_{\mathcal{L}(X, X)} \leq c_{8} h K \text {. }
$$

Obviously,

$$
D_{2} F^{h}\left(\lambda, \underline{\hat{\mathbf{u}}}_{h, \lambda}\right)=D_{2} F\left(\lambda, \underline{\mathbf{u}}_{\lambda}\right)\left[I-D_{2} F\left(\lambda, \underline{\mathbf{u}}_{\lambda}\right)^{-1}\left(D_{2} F\left(\lambda, \underline{\mathbf{u}}_{\lambda}\right)-D_{2} F^{h}\left(\lambda, \underline{\hat{\mathbf{u}}}_{h, \lambda}\right)\right)\right] .
$$

Because of (6.10) we have, for $0<h \leq h_{1}:=1 / 2 \gamma^{*} c_{8} K$,

$$
\left\|D_{2} F\left(\lambda, \underline{\mathbf{u}}_{\lambda}\right)^{-1}\left(D_{2} F\left(\lambda, \underline{\mathbf{u}}_{\lambda}\right)-D_{2} F^{h}\left(\lambda, \underline{\hat{\mathbf{u}}}_{h, \lambda}\right)\right)\right\|_{\mathcal{L}(X, X)} \leq \gamma(\lambda) c_{8} h K \leq \frac{1}{2} .
$$

Then we conclude that $I-D_{2} F\left(\lambda, \underline{\mathbf{u}}_{\lambda}\right)^{-1}\left(D_{2} F\left(\lambda, \underline{\mathbf{u}}_{\lambda}\right)-D_{2} F^{h}\left(\lambda, \underline{\hat{\mathbf{u}}}_{h, \lambda}\right)\right)$ is an isomorphism with norm of its inverse $\leq 2$. Taking (6.11) into account, we obtain that $D_{2} F^{h}\left(\lambda, \underline{\hat{\mathbf{u}}}_{h, \lambda}\right)$ is an isomorphism with

$$
\left\|D_{2} F^{h}\left(\lambda, \underline{\hat{\mathbf{u}}}_{h, \lambda}\right)^{-1}\right\|_{\mathcal{L}(X, X)} \leq 2 \gamma(\lambda) .
$$

Analogous arguments yield, for $\underline{\mathbf{v}}_{i}:=\left(\left(\mathbf{v}_{i}, \boldsymbol{\Psi}_{i}\right), \chi_{i}\right) \in X, i=1,2$, and $\underline{\mathbf{w}} \in X$ with $\|\underline{\mathbf{w}}\|_{X}=1$ as above,

$$
\begin{aligned}
& \left\|D_{2} F^{h}\left(\lambda, \underline{\mathbf{v}}_{1}\right) \underline{\mathbf{w}}-D_{2} F^{h}\left(\lambda, \underline{\mathbf{v}}_{2}\right) \underline{\mathbf{w}}\right\|_{X} \\
& \quad=\left\|T^{h} D_{2} G^{h}\left(\lambda, \underline{\mathbf{v}}_{1}\right) \underline{\mathbf{w}}-T^{h} D_{2} G^{h}\left(\lambda, \underline{\mathbf{v}}_{2}\right) \underline{\mathbf{w}}\right\|_{X} \\
& \quad \leq c_{9}\left\|\underline{\mathbf{v}}_{1}-\underline{\mathbf{v}}_{2}\right\|_{X} .
\end{aligned}
$$


Take $0<h \leq h_{1}$. For $\underline{\mathbf{v}}_{h}:=\left(\left(\mathbf{v}_{h}, \mathbf{\Psi}_{h}\right), \chi_{h}\right) \in X^{h}$ we define

$$
\mathcal{F}: X^{h} \rightarrow X^{h}, \quad \mathcal{F}\left(\underline{\mathbf{v}}_{h}\right):=\underline{\mathbf{v}}_{h}-D_{2} F^{h}\left(\lambda, \underline{\hat{\mathbf{u}}}_{h, \lambda}\right)^{-1} F^{h}\left(\lambda, \underline{\mathbf{v}}_{h}\right) .
$$

It is obvious that we have a fixed point of $\mathcal{F}$ if and only if it is a solution of the operator equation (6.7).

From (6.9), (6.12) and (6.13) we conclude the existence of a real $h_{0}>0$ such that for each $0<h \leq h_{0}$ the map $\mathcal{F}$ is a contraction on the set $B\left(\underline{\hat{\underline{u}}}_{h, \lambda}, R\right)$ with $R=R(h, \lambda)$. Consequently, applying Banach's fixed point theorem, we obtain the existence of a unique solution $\underline{\mathbf{u}}_{h, \lambda}:=\left(\left(\mathbf{u}_{h, \lambda}, \mathbf{B}_{h, \lambda}\right), p_{h, \lambda}\right) \in B\left(\underline{\hat{\mathbf{u}}}_{h, \lambda}, R\right)$ of problem (6.7). The error estimate (6.8) follows with the triangle inequality and interpolation estimates (see [11]).

\section{REFERENCES}

[1] R. A. Adams, Sobolev Spaces, Academic Press, New York, 1975. MR 56:9247

[2] F. Brezzi, J. Rappaz and P.-A. Raviart, Finite-dimensional approximation of nonlinear problems. Part I: Branches of nonsingular solutions, Numer. Math. 36 (1980), 1-25. MR 83f: $65089 \mathrm{a}$

[3] P. G. Ciarlet, The Finite Element Method for Elliptic Problems, North-Holland, Amsterdam, 1978. MR 58:25001

[4] P. Clément, Approximation by finite element functions using local regularization, RAIRO Anal. Numér. 9 (1975), 77-84. MR 53:4569

[5] V. Girault and P.-A. Raviart, Finite Element Methods for Navier-Stokes Equations. Theory and Algorithms, Springer-Verlag, Berlin, 1986. MR 88b:65129

[6] R. Glowinski, Numerical Methods for Nonlinear Variational Problems, Springer-Verlag, New York (1984). MR 86c:65004

[7] P. Grisvard, Elliptic Problems in Nonsmooth Domains, Pitman, Boston, 1985. MR 86m:35044

[8] M. D. Gunzburger, Finite Element Methods for Viscous Incompressible Flows, Academic Press, Boston, 1989. MR 91d:76053

[9] M. D. Gunzburger, A. J. Meir, and J. S. Peterson, On the existence, uniqueness, and finite element approximation of solutions of the equations of stationary, incompressible magnetohydrodynamics, Math. Comp. 56 (1991), 523-563. MR 91m:76127

[10] G. H. Hardy, J. E. Littlewood and G. Polya, Inequalities, Cambridge University Press, Cambridge, 1952. MR 13:727e

[11] L. Tobiska, and R. Verfr̈uth, Analysis of a streamline diffusion finite element method for the Stokes and Navier-Stokes equations, SIAM J. Numer. Anal. 33 (1996), 107-127. MR 97e:65133

[12] M. Wiedmer, Finite-Elemente-Approximation für Gleichungen aus der Magnetohydrodynamik, Dissertation, Ruhr-Universität Bochum (1997).

Fakultät für Mathematik, Ruhr-Universität Bochum, D-44780 Bochum, Germany Current address: Usterstrasse 29, CH-8620 Wetzikon, Switzerland

E-mail address: wiedmer@smile.ch 